HD-THEP-08-9

SIAS-CMTP-08-1

CPHT-RR002.0108

LPT-ORSAY-08-16

\title{
Compact heterotic orbifolds in blow-up
}

\author{
Stefan Groot Nibbelink ${ }^{a}$, Denis Klevers ${ }^{b}$, Felix Plöger ${ }^{b, c}$, \\ Michele Trapletti ${ }^{d}$, Patrick K.S. Vaudrevange ${ }^{b}$ \\ ${ }^{a}$ Institut für Theoretische Physik, Universität Heidelberg, Philosophenweg 16 und 19, \\ D-69120 Heidelberg, Germany 83 Shanghai Institute for Advanced Study, University of \\ Science and Technology of China, 99 Xiupu Rd, Pudong, Shanghai 201315, P.R. China \\ ${ }^{b}$ Physikalisches Institut der Universität Bonn, Nussallee 12, D-53115 Bonn, Germany \\ ${ }^{c}$ Institut für Chemie und Dynamik der Geosphäre, ICG-1: Stratosphäre, \\ Forschungszentrum Jülich GmbH, D-52425 Jülich, Germany \\ ${ }^{d}$ Laboratoire de Physique Theorique, Bat. 210, Université de Paris-Sud, F-91405 \\ Orsay, France \& Centre de Physique Théorique, École Polytechnique, F-91128 \\ Palaiseau, France
}

\begin{abstract}
We compare heterotic string models on orbifolds with supergravity models on smooth compact spaces, obtained by resolving the orbifold singularities. Our main focus is on heterotic $\mathrm{E}_{8} \times \mathrm{E}_{8}^{\prime}$ models on the resolution of the compact $T^{6} / \mathbb{Z}_{3}$ orbifold with Wilson lines. We explain how different gauge fluxes at various resolved fixed points can be interpreted in blow down as Wilson lines. Even when such Wilson lines are trivial from the orbifold perspective, they can still lead to additional symmetry breaking in blow-up. Full agreement is achieved between orbifold and resolved models, at the level of gauge interactions, massless spectrum and anomaly cancellation. In this matching the blow-up modes are of crucial importance: they play the role of model-dependent axions involved in the cancellation of multiple anomalous $\mathrm{U}(1)$ 's on the resolution. We illustrate various aspects by investigating blow-ups of a $\mathbb{Z}_{3}$ MSSM model with two Wilson lines: if all its fixed points are resolved simultaneously, the SM gauge group is necessarily broken. Finally, we explore in detail the anomaly cancellation on the complex two dimensional resolution of $\mathbb{C}^{2} / \mathbb{Z}_{2}$.
\end{abstract}




\section{Introduction}

Orbifold compactification of the heterotic string [1] has been one of the first approaches to string phenomenology. In the past, vast scans of possible 4D models were undertaken with the aim of reproducing the spectrum and the interactions of the Standard Model of particle physics or of a supersymmetric extension of it (MSSM), see e.g. [2]. The interest in this approach has been recently revived with the initial goal to obtain "orbifold GUTs" [3] from string compactifications [4, 5, 6, 7, 8]. With this technology in mind, the original aim of building a 4D MSSM model was re-established, leading to many successful constructions [7, 9] that are nowadays some of the best known string models (for other constructions see e.g. [10, 11, 12, 13]).

The orbifold constructions have proven to be one of the most successful approaches to string phenomenology, yet this approach has a severe limitation: exact string quantization is only possible on the orbifold, as it is constructed by combining free conformal field theories (CFTs). This means that "calculability" is limited to a single point in the moduli space of the model. This does not mean that away from the orbifold point one has no control over the resulting 4D model: we can describe a model away from the orbifold point by giving vevs to some twisted states. Nevertheless, this extrapolation away from the orbifold point in moduli space is sensible only if these vevs are sufficiently small. Otherwise, the standard truncation to the 4D supergravity Lagrangian cannot be trusted. However, there are good reasons to consider big deformations of the orbifold model: having access to only a limited region in moduli space makes it virtually impossible to achieve an efficient moduli stabilization mechanism or to study supersymmetry breaking vacua.

In order to overcome this obstacle, it would be crucial to combine the model building power of orbifold constructions with an approach able to characterize realistic models away from the orbifold point in moduli space, i.e. when the orbifold singularities are resolved [14]. This is the main intention of this paper. To do so, we build on the results of [15], where the resolutions of $\mathbb{C}^{n} / \mathbb{Z}_{n}$ singularities were considered. The freedom in the embedding of the $\mathrm{U}(1)$ bundle on these resolution spaces into the $\mathrm{SO}(32)$ gauge group of 10D heterotic supergravity allowed for the construction of a range of resolved models in 4D and 6D. These models could be matched with the corresponding singular orbifold models built by quantizing the heterotic string on $\mathbb{C}^{n} / \mathbb{Z}_{n}$, with $n=2,3$, using the standard CFT techniques. In this matching it was crucial to "blow-up" the orbifold model by giving a vev to a certain twisted scalar which defines the "blow-up mode" [16]. This matching was refined in [17, where the issues of multiple anomalous U(1)'s and generalized Green-Schwarz mechanisms were addressed. Using toric geometry [18] this program can be carried out to a much wider class of orbifold singularities.

In the present paper we want to consider the $\mathrm{E}_{8} \times \mathrm{E}_{8}^{\prime}$ heterotic string on the compact orbifold $T^{6} / \mathbb{Z}_{3}$. (For a discussion of the heterotic $\mathrm{SO}(32)$ string on such an orbifold, see e.g. [19].) The compactness of this orbifold is very relevant for phenomenology since, apart from a finite 4D Planck mass, it allows us to include discrete Wilson lines, which are crucial for model building. To prepare for our study of this compact orbifold, we 
extend the results of [15] to the $\mathrm{E}_{8} \times \mathrm{E}_{8}^{\prime}$ heterotic string on $\mathbb{C}^{3} / \mathbb{Z}_{3}$ in Section 2. We first recall the resolved geometry and the form of $\mathrm{U}(1)$ bundles on it. After this we consider all possible $\mathrm{E}_{8} \times \mathrm{E}_{8}^{\prime}$ embeddings of the $\mathrm{U}(1)$ flux and describe the resulting five inequivalent resolved models. We relate each resolution model to a known heterotic orbifold model by switching on a certain blow-up mode. We check that the vev of this twisted state is essentially compatible with F- and D-flatness. Finally, we explain how one can use field redefinitions to understand that their spectra agree in detail.

In Section 3 we construct the resolution of the compact $T^{6} / \mathbb{Z}_{3}$ orbifold by cutting a local patch around each singularity and replacing it by the resolved space with $\mathrm{U}(1)$ bundles, as described in Section 2. The matching in the absence of discrete Wilson lines seems to be a straightforward extension of the results of Section 2 . However, two minor complications arise: firstly, the superpotential in the compact case is generically more complicated than the non-compact one, hence F-flatness needs to be rechecked. Secondly, it is possible that there is a trivial Wilson line between two orbifold fixed points, which in blow-up nevertheless leads to a further symmetry breaking.

From the resolution perspective, we interpret discrete Wilson lines as the possibility of wrapping different fluxes around each resolved singularity. In other words, this freedom can be understood as non-trivial transition functions for the gauge backgrounds going from one resolved singularity to the other. We study the consistency conditions for the transition functions. Furthermore, we show that the presence of these transition functions affects the computation of the unbroken 4D gauge group and of the localized (twisted) and delocalized (untwisted) matter spectrum. We conclude this section with two examples: the first example illustrates how to embed a discrete Wilson line on the resolution of $T^{6} / \mathbb{Z}_{3}$ and exemplifies the possibility of having multiple anomalous U(1)'s in compact blow-ups. The second example demonstrates some of the potential consequences of blowing up all singularities for semi-realistic MSSM-like models: contrary to the orbifold theory, in a full resolution of the model in exam the hypercharge $U(1)$ is necessarily broken.

In Section 4 we pass to the study of resolutions of the $\mathbb{C}^{2} / \mathbb{Z}_{2}$ orbifold and extend the purely topological approach to the resolution of $T^{4} / \mathbb{Z}_{2}$ singularities, as considered in [20]: there it was noted that the 6D anomaly polynomials of the heterotic orbifold and of the related smooth models are not the same. We analyze this problem in the same spirit of Section 2, matching the models at the level of the gauge interactions and spectra after giving a vev to a suitable blow-up mode. We show that the matching of the anomaly cancellations requires carefully considering the consequences of the field redefinitions that make the $\mathrm{U}(1)$ charges of the models match.

The paper is concluded by Section 5, which summarizes our main findings.

\section{Heterotic $\mathbb{C}^{3} / \mathbb{Z}_{3}$ orbifold and resolution models}

We consider the heterotic string quantized on the singular space $\mathbb{M}^{4} \times \mathbb{C}^{3} / \mathbb{Z}_{3}$ and on its resolution. We start by giving the geometrical details of the $\mathbb{C}^{3} / \mathbb{Z}_{3}$ singularity. Then 
we show how to resolve it and how to construct gauge fluxes on the resolution. After this study of the geometry, we consider the heterotic string on the singular space and on the resolution, leading to $4 \mathrm{D}$ heterotic orbifold and resolved models, respectively. Finally, we investigate how the two classes of models match with particular care for the anomaly cancellation: we show that, on the orbifold side, the standard Green-Schwarz mechanism, involving one single universal axion, is combined with a Higgs mechanism giving rise to the blow-up. On the resolution, this combination is mapped into a GreenSchwarz mechanism involving two axions. These are mixtures of the orbifold axion and of the blow-up mode. This identification is completed by the observation that the new Fayet-Iliopoulos term produced on the resolution is nothing else than the (tree-level) D-term due to the non-vanishing vev of the blow-up mode.

\subsection{Orbifold and blow-up geometry}

We start from $\mathbb{C}^{3}$ parameterized by the three complex coordinates $\tilde{Z}^{A}(A=1,2,3)$, on which the orbifold rotation $\Theta$ acts as

$$
\Theta: \tilde{Z}^{A} \longmapsto e^{2 \pi i \phi_{A} / 3} \tilde{Z}^{A}, \quad \phi=(1,1,-2) .
$$

The non-compact orbifold $\mathbb{C}^{3} / \mathbb{Z}_{3}$ is obtained by identifying those points in $\mathbb{C}^{3}$ that are mapped into each other by $\Theta$. Such a space is singular in the fixed point $\{0\}$, and is naturally equipped with a Kähler potential, inherited from $\mathbb{C}^{3}$,

$$
\mathcal{K}_{\mathbb{C}^{3} / \mathbb{Z}_{3}}=\sum_{A} \overline{\tilde{Z}}^{A} \tilde{Z}^{A}
$$

We can cover $\mathbb{C}^{3} / \mathbb{Z}_{3}-\{0\}$ by means of three coordinate patches, defined as

$$
U_{(A)} \equiv\left\{\tilde{Z} \in \mathbb{C}^{3} \mid \tilde{Z}^{A} \neq 0,0<\arg \left(\tilde{Z}^{A}\right)<2 \pi / 3\right\}, \quad A=1,2,3 .
$$

It is convenient to choose new coordinates on the orbifold, which allow for a systematic construction of a resolution of the singularity as a line bundle over $\mathbb{C} \mathbb{P}^{2}$. In the language of toric geometry [18, 21], the $\mathbb{C P}^{2}$ is called an exceptional divisor, and it replaces the singularity in the resolution $\mathcal{M}^{3}$ of $\mathbb{C}^{3} / \mathbb{Z}_{3}$. When its volume shrinks to zero, the singularity is recovered, and the space $\mathcal{M}^{3}$ approaches $\mathbb{C}^{3} / \mathbb{Z}_{3}$ (blow-down). Thus the blowing-up/down procedure is controlled by the size of the exceptional divisor. To make this more explicit we consider the patch $U_{(A)}$, where $\tilde{Z}^{A} \neq 0$, and define $z^{B} \equiv \tilde{Z}^{B} / \tilde{Z}^{A}$ for $B \neq A$. To remove the deficit angle of $\tilde{Z}^{A}$ we perform the coordinate transformation $\tilde{Z}^{A} \mapsto x \equiv\left(\tilde{Z}^{A}\right)^{3}$. In this way the Kähler potential becomes

$$
\mathcal{K}_{\mathbb{C}^{3} / \mathbb{Z}_{3}}=X^{\frac{1}{3}}, \quad X \equiv \bar{x}(1+\bar{z} z)^{3} x .
$$

This change of variables trades the deficit angle for a non-analyticity in $\mathcal{K}_{\mathbb{C}^{3} / \mathbb{Z}_{3}}$.

A resolution $\mathcal{M}^{3}$ of the orbifold is given by considering the open patches introduced above, equipped with a new Kähler potential [15]

$$
\mathcal{K}_{\mathcal{M}^{3}}=\int_{1}^{X} \frac{\mathrm{d} X^{\prime}}{X^{\prime}} M\left(X^{\prime}\right), \quad M(X)=\frac{1}{3}(r+X)^{\frac{1}{3}}
$$




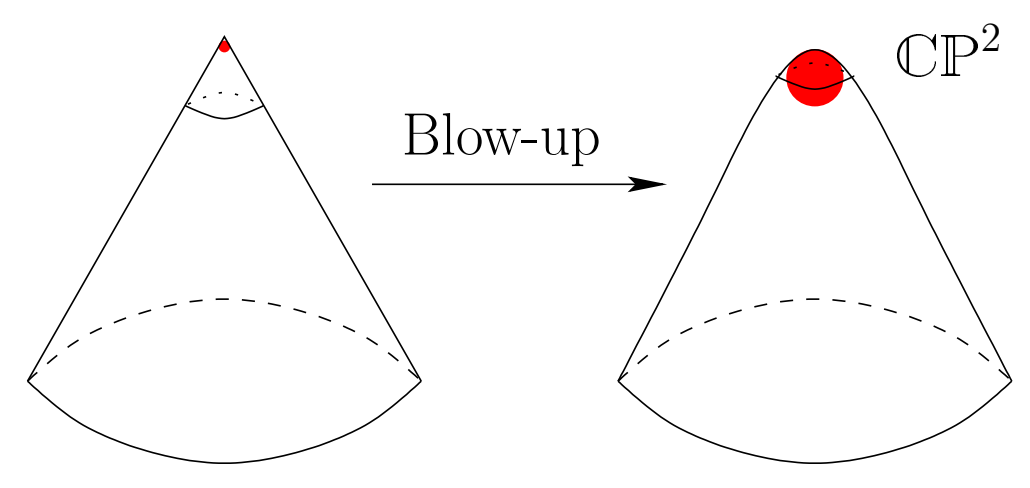

Figure 1: The orbifold singularity is cut out locally and a smooth resolution manifold (containing an exceptional divisor $\mathbb{C P}^{2}$ ) glued in.

that is Ricci-flat and matches the orbifold Kähler potential in the $r \rightarrow 0$ limit. In this limit the curvature vanishes for points where $x \neq 0$, whereas for $x=0$ it diverges. Moreover, it vanishes for any value of $r$ when $|x| \rightarrow \infty$. Therefore, blowing up means that the orbifold singularity is replaced by the smooth compact $\mathbb{C P}^{2}$ that shrinks to zero as $r \rightarrow 0$ (the situation is illustrated in Figure 1).

\subsection{Gauge fluxes wrapped on the orbifold and the resolution}

When defining the heterotic string on $\mathbb{C}^{3} / \mathbb{Z}_{3}$, the $10 \mathrm{D}$ gauge group $\mathrm{E}_{8} \times \mathrm{E}_{8}^{\prime} 1$ is broken by the orbifolding procedure. We can understand this breaking from an effective field theory perspective: let $i \mathfrak{A}$ be the one-form gauge field, with values in $\mathrm{E}_{8} \times \mathrm{E}_{8}^{\prime}$, and let $i \mathfrak{F}$ be its field strength. Moreover, define $H_{I}$, for $I=1, \ldots, 16$, as the basis elements of the Cartan subalgebra of $\mathrm{E}_{8} \times \mathrm{E}_{8}^{\prime}$. In a given coordinate patch with local coordinates $z, x=|x| e^{i \phi}$, the orbifold action $\Theta$ is realized as $\phi \rightarrow \phi+2 \pi$. On the orbifold there can be non-trivial orbifold boundary conditions for $\mathfrak{A}$

$$
i \mathfrak{A}(\Theta \tilde{Z})=i \mathfrak{A}(z,|x|, \phi+2 \pi)=U i \mathfrak{A}(z,|x|, \phi) U^{-1},
$$

where $U=e^{2 \pi i\left(V_{\text {orb }}^{I} H_{I}\right) / 3}$ and $V_{\text {orb }}$ is a vector in the $\mathrm{E}_{8} \times \mathrm{E}_{8}^{\prime}$ root lattice. These boundary conditions induce a gauge symmetry breaking, precisely to those $\mathrm{E}_{8} \times \mathrm{E}_{8}^{\prime}$ algebra elements with root vectors $p$ such that $p \cdot V_{\text {orb }}=0 \bmod 3$. Our normalization of the orbifold gauge shift vector $V_{\text {orb }}$ differs by a factor $1 / 3$ from the common one; our convention avoids such an additional factor when we make identification with gauge bundle fluxes below.

The non-trivial orbifold boundary conditions can be reformulated in terms of fields with trivial ones, but having a non-zero constant gauge background. The existence of this non-vanishing gauge flux, localized at the singularity, should become "visible" on the resolution. To obtain a matching of orbifold models with models built on the resolved space, we consider the possibility of a gauge bundle wrapped around the resolution. In general such a bundle has structure group $\mathcal{J}$ embedded into $\mathrm{E}_{8} \times \mathrm{E}_{8}^{\prime}$. This embedding

\footnotetext{
${ }^{1}$ We restrict to this case as the $\mathrm{SO}(32)$ theory was considered in [15, 17.
} 
breaks the $10 \mathrm{D}$ gauge group $\mathrm{E}_{8} \times \mathrm{E}_{8}^{\prime}$ to the maximal subgroup $H \subset \mathrm{E}_{8} \times \mathrm{E}_{8}^{\prime}$ that commutes with $\mathcal{J}$. We therefore expand the $10 \mathrm{D}$ gauge field strength $i \mathfrak{F}=i \mathcal{F}+i F$ around the internal background $i \mathcal{F}$, living in the algebra of $\mathcal{J}$, in terms of the $4 \mathrm{D}$ gauge field strength $i F$, taking values in the algebra of $H$. To preserve $N=1$ supersymmetry in four dimensions, the bundle field strength has to satisfy the Hermitian Yang-Mills equations $[22]^{2}$

$$
\mathcal{F}_{A B}=0, \quad \mathcal{F}_{\bar{A} \bar{B}}=0, \quad G^{A \bar{A}} \mathcal{F}_{A \bar{A}}=0,
$$

where $G^{A \bar{A}}$ denotes the inverse Hermitian metric of $\mathcal{M}^{3}$. One further (topological) consistency requirement follows from the integrated Bianchi-identity of the two-form $B$ of the supergravity multiplet:

$$
\int_{\mathcal{C}^{4}}\left(\operatorname{tr} \mathcal{R}^{2}-\operatorname{tr}(i \mathcal{F})^{2}\right)=0
$$

for all compact four-cycles $\mathcal{C}^{4}$ of the resolution and $\mathcal{R}$ denotes the curvature of the internal space $\mathcal{M}^{3}$. This condition is crucial to ensure that the effective four dimensional theory is free of non-Abelian anomalies [24. The resolution space $\mathcal{M}^{3}$ only contains a single compact four-cycle, the $\mathbb{C P}^{2}$ at the resolved singularity, leading to a single consistency condition.

We give two examples of bundles on the resolution that satisfy (7) and (8). The simplest construction of such a bundle is the standard embedding (to which we refer as "AS") with the gauge connection taken to be equal to the spin connection [22]. In terms of the curvature this means $i \mathcal{F}=\mathcal{R}$. Since $\mathcal{R} \in \mathrm{SU}(3)$, this describes an $\mathrm{SU}(3)$ bundle, embedded into $\mathrm{E}_{8} \times \mathrm{E}_{8}^{\prime}$, leading to the $4 \mathrm{D}$ gauge group $H=\mathrm{E}_{6} \times \mathrm{E}_{8}^{\prime}$. However, in this paper we mainly focus on the $\mathrm{U}(1)$ gauge bundle with field strength

$$
i \mathcal{F}=\left(\frac{r}{r+X}\right)^{1-\frac{1}{n}}\left(\bar{e} e-\frac{n-1}{n^{2}} \frac{1}{r+X} \bar{\epsilon} \epsilon\right),
$$

see [15] for notational conventions. Such a bundle can be embedded into $\mathrm{E}_{8} \times \mathrm{E}_{8}^{\prime}$ as

$$
i \mathcal{F}_{V}=i \mathcal{F} H_{V}
$$

where we use the notation $H_{V} \equiv V^{I} H_{I}$. Since the bundle is only well-defined if its first Chern class, integrated over all compact two-cycles, is integral, an extra consistency requirement arises for the vector $V^{I}$. For the two-cycle $\mathbb{C P}^{1}$ at $x=0$,

$$
\frac{1}{2 \pi i} \int_{\mathbb{C P}^{1}} i \mathcal{F}_{V}=V^{I} H_{I}
$$

must be integral for all $\mathrm{E}_{8} \times \mathrm{E}_{8}^{\prime}$ roots. This implies that $V$ has to be an $\mathrm{E}_{8} \times \mathrm{E}_{8}^{\prime}$ root lattice vector itself. The two-form $\mathcal{F}$ is regular everywhere for $r \neq 0$. In the blow-down limit $r \rightarrow 0$, it is zero for $x \neq 0$ and it diverges for $x=0$, in such a way that the integral above remains constant. This means that the bundle is "visible" as a two-form only in the blow-up, but in the blow-down its physical effect is not lost because the gauge flux gets localized in the fixed point. In this sense, this bundle is exactly the counterpart of the orbifold boundary conditions discussed above.

\footnotetext{
${ }^{2}$ Here we ignore loop corrections to these equations, discussed in [23. We will return to this point later in the paper.
} 


\begin{tabular}{|c|c|c|}
\hline$V_{\text {orb }}$ & gauge group & \\
\hline \hline$\left(0^{8}\right)\left(0^{8}\right)$ & $\mathrm{E}_{8} \times \mathrm{E}_{8}^{\prime}$ & \\
$\left(-2,1^{2}, 0^{5}\right)\left(0^{8}\right)$ & $\mathrm{E}_{6} \times \mathrm{SU}(3) \times \mathrm{E}_{8}^{\prime}$ & $\mathrm{A}$ \\
$\left(-2,1^{2}, 0^{5}\right)\left(-2,1^{2}, 0^{5}\right)$ & {$\left[\mathrm{E}_{6} \times \mathrm{SU}(3)\right]^{2}$} & $\mathrm{~B}$ \\
$\left(1^{2}, 0^{6}\right)\left(-2,0^{7}\right)$ & $\mathrm{E}_{7} \times \mathrm{SO}(14) \times \mathrm{U}(1)^{2}$ & $\mathrm{C}$ \\
$\left(-2,1^{4}, 0^{3}\right)\left(-2,0^{7}\right)$ & $\mathrm{SU}(9) \times \mathrm{SO}(14) \times \mathrm{U}(1)$ & $\mathrm{D}$ \\
\hline
\end{tabular}

Table 1: We list all inequivalent $\mathbb{C}^{3} / \mathbb{Z}_{3}$ orbifold shifts and the corresponding gauge symmetry breakings. For shifts, expressions like $0^{n}$ denote $n$ times the zero entry.

\subsection{Classifying orbifold and smooth line bundle models}

The heterotic string on the $\mathbb{C}^{3} / \mathbb{Z}_{3}$ is specified by the orbifold gauge shift vector $V_{\text {orb }}$ defined in (6). The freedom in the choice of $V_{\text {orb }}$ is constrained by modular invariance of the string partition function:

$$
V_{\text {orb }}^{2}=0 \bmod 6 .
$$

There are only five inequivalent shift vectors, each of them giving rise to a different orbifold model. In Table 1 we list the possible $V_{\text {orb }}$ together with the gauge groups surviving the orbifold projection. Using the standard CFT procedure it is possible to compute the spectra of these models. They are listed in the second column of Table 3 . The spectra are given with the multiplicity numbers with which the various states contribute to the $4 \mathrm{D}$ anomaly polynomial localized in the singularity. Thus, these numbers can be fractional if the corresponding states are not localized in the $\mathbb{C}^{3} / \mathbb{Z}_{3}$ singularity. The untwisted states have multiplicities that are multiples of $3 / 27$, because the compact orbifold $T^{6} / \mathbb{Z}_{3}$ has 27 singularities and untwisted states come with multiplicity three. On the other hand, these multiplicities are integers for localized (i.e. twisted) states.

The blow-up model is completely specified by the way how the gauge flux is embedded in $\mathrm{E}_{8} \times \mathrm{E}_{8}^{\prime}$, i.e. by the vector $V$. The Bianchi identity integrated over $\mathbb{C P}^{2}$ yields the consistency condition

$$
V^{2}=12
$$

and enormously constrains the number of possible models. All solutions to (13) together with the corresponding gauge groups are given in Table 2. The chiral matter content is determined by the Dirac index theorem that for $\mathrm{U}(1)$ bundles takes the form of a multiplicity operator

$$
N_{V}=\frac{1}{18}\left(H_{V}\right)^{3}-\frac{1}{6} H_{V}
$$

see [15] for details. The computation of the spectra for each of the U(1) embeddings shows that there are in fact only five inequivalent models amongst them. We distinguish them by their chiral spectra, which are given in the third column of Table 3 . 


\begin{tabular}{|c|c|c|c|c|}
\hline & $\begin{array}{l}\text { bundle vector } \\
V=\left(V_{1}\right)\left(V_{2}\right)\end{array}$ & & $\begin{array}{c}\text { gauge group } \\
V_{1}^{2}+V_{2}^{2}=V^{2}=12\end{array}$ & label \\
\hline $\begin{array}{l}\left(3,1^{3}, 0^{4}\right)\left(0^{8}\right) \\
\left(\frac{5}{2}, \frac{3}{2}^{2}, \frac{1}{2}^{5}\right)\left(0^{8}\right)\end{array}$ & $\begin{array}{c}\left(2^{3}, 0^{5}\right)\left(0^{8}\right) \\
\left(\frac{3}{2}^{4},-\frac{3}{2}, \frac{1}{2}^{3}\right)\left(0^{8}\right)\end{array}$ & $\left(2^{2}, 1^{4}, 0^{2}\right)\left(0^{8}\right)$ & $\begin{array}{c}\mathrm{SO}(10) \times \mathrm{U}(3) \times \mathrm{E}_{8}^{\prime} \\
12+0\end{array}$ & $\mathrm{AI}$ \\
\hline $\begin{array}{c}\left(2,1^{2}, 0^{5}\right)\left(2,1^{2}, 0^{5}\right) \\
\left(1^{6}, 0^{2}\right)\left(1^{6}, 0^{2}\right)\end{array}$ & $\begin{array}{l}\left(2,1^{2}, 0^{5}\right)\left(1^{6}, 0^{2}\right) \\
\left(1^{6}, 0^{2}\right)\left(\frac{3}{2}^{2}, \frac{1}{2}^{6}\right)\end{array}$ & $\begin{array}{l}\left(2,1^{2}, 0^{5}\right)\left(\frac{3}{2}^{2}, \frac{1}{2}^{6}\right) \\
\left(\frac{3}{2}^{2}, \frac{1}{2}^{6}\right)\left(\frac{3}{2}^{2}, \frac{1}{2}^{6}\right)\end{array}$ & $\begin{array}{c}\left(\mathrm{E}_{6} \times \mathrm{U}(2)\right)^{2} \\
6+6\end{array}$ & $\mathrm{BI}$ \\
\hline $\begin{array}{c}\left(2^{2}, 0^{6}\right)\left(2,0^{7}\right) \\
\left(1^{8}\right)\left(2,0^{7}\right)\end{array}$ & $\begin{array}{c}\left(2^{2}, 0^{6}\right)\left(1^{4}, 0^{4}\right) \\
\left(1^{8}\right)\left(1^{4}, 0^{4}\right)\end{array}$ & $\begin{array}{c}\left(2^{2}, 0^{6}\right)\left(-\frac{3}{2}, \frac{1}{2}^{7}\right) \\
\left(1^{8}\right)\left(-\frac{3}{2}, \frac{1}{2}^{7}\right)\end{array}$ & $\begin{array}{c}\mathrm{E}_{7} \times \mathrm{SO}(14)^{\prime} \times \mathrm{U}(1)^{2} \\
8+4\end{array}$ & $\mathrm{CI}$ \\
\hline $\begin{array}{c}\left(1^{2}, 0^{6}\right)\left(3,1,0^{6}\right) \\
\left(1^{2}, 0^{6}\right)\left(\frac{5}{2},-\frac{3}{2}, \frac{1}{2}^{6}\right) \\
\left(\frac{1}{2}^{8}\right)\left(2^{2}, 1^{2}, 0^{4}\right) \\
\left(\frac{1}{2}^{8}\right)\left(\frac{3}{2}^{4}, \frac{1}{2}^{4}\right)\end{array}$ & $\begin{array}{c}\left(1^{2}, 0^{6}\right)\left(2^{2}, 1^{2}, 0^{4}\right) \\
\left(1^{2}, 0^{6}\right)\left(\frac{3}{2}^{4}, \frac{1}{2}^{4}\right) \\
\left(\frac{1}{2}^{8}\right)\left(2,1^{6}, 0\right)\end{array}$ & $\begin{array}{c}\left(1^{2}, 0^{6}\right)\left(2,1^{6}, 0\right) \\
\left(\frac{1}{2}^{8}\right)\left(3,1,0^{6}\right) \\
\left(\frac{1}{2}^{8}\right)\left(\frac{5}{2},-\frac{3}{2}, \frac{1}{2}^{6}\right)\end{array}$ & $\begin{array}{c}\mathrm{E}_{7} \times \mathrm{SO}(12)^{\prime} \times \mathrm{U}(1)^{3} \\
2+10\end{array}$ & CII \\
\hline $\begin{array}{c}\left(2,1^{4}, 0^{3}\right)\left(2,0^{7}\right) \\
\left(-\frac{3}{2}^{3}, \frac{1}{2}^{5}\right)\left(2,0^{7}\right) \\
\left(\frac{5}{2}, \frac{1}{2}^{7}\right)\left(2,0^{7}\right) \\
\left(-1,1^{7}\right)\left(2,0^{7}\right)\end{array}$ & $\begin{array}{c}\left(2,1^{4}, 0^{3}\right)\left(1^{4}, 0^{4}\right) \\
\left(-\frac{3}{2}^{3}, \frac{1}{2}^{5}\right)\left(1^{4}, 0^{4}\right) \\
\left(\frac{5}{2}, \frac{1}{2}^{7}\right)\left(1^{4}, 0^{4}\right) \\
\left(-1,1^{7}\right)\left(1^{4}, 0^{4}\right)\end{array}$ & $\begin{array}{c}\left(2,1^{4}, 0^{3}\right)\left(-\frac{3}{2}, \frac{1}{2}^{7}\right) \\
\left(-\frac{3}{2}^{3}, \frac{1}{2}^{5}\right)\left(-\frac{3}{2}^{2}, \frac{1}{2}^{7}\right) \\
\left(\frac{5}{2}, \frac{1}{2}^{7}\right)\left(-\frac{3}{2}^{2}, \frac{1}{2}^{7}\right) \\
\left(-1,1^{7}\right)\left(-\frac{3}{2}, \frac{1}{2}^{7}\right)\end{array}$ & $\begin{array}{c}\mathrm{SU}(8) \times \mathrm{SO}(14)^{\prime} \times \mathrm{U}(1)^{2} \\
8+4\end{array}$ & DI \\
\hline
\end{tabular}

Table 2: In this table we list all consistent $\mathrm{U}(1)$ bundles embedded into $\mathrm{E}_{8} \times \mathrm{E}_{8}^{\prime}$. We group together the embeddings characterized by the bundle vector $V$ producing the same gauge symmetry breaking and localized spectrum. Each group corresponds to a distinct blow-up of the orbifold models. The bundle vector $V$ contains two parts corresponding to both $\mathrm{E}_{8}$ 's. Most models are characterized by the values of $V_{1}^{2}$ and $V_{2}^{2}$; only the splitting $8+4$ has two realizations.

\subsection{Matching orbifold and blow-up models}

We now want to investigate the matching between the heterotic orbifold and the blowup models discussed in the previous section. This matching can be considered at various levels and we begin with some simple observations before entering more subtle issues.

The first basic observation was made in (6) : the embedding of the orbifold rotation in the gauge bundle (via the shift $V_{\text {orb }}$ ) can be seen as the presence of a gauge flux localized in the singularity. On the resolution, such a gauge flux appears, and it is immediate to identify

$$
\frac{1}{3} V^{I} H_{I}=\frac{1}{2 \pi i} \int_{\mathbb{C}} i \mathcal{F}_{V} \rightarrow \frac{1}{3} V_{\text {orb }}^{I} H_{I} .
$$

The integration above is made on the variable $x$ defined in (6) in such a way that the integral can be immediately read as a contour integral of $\mathcal{A}$ around the phase $\phi$ of $x$ or, in other words, precisely as the Wilson line associated with $V_{\text {orb }}^{I} H_{I}$ in (6) .

This basic observation is corroborated by the fact that any blow-up shift $V$, listed in Table 2, is modular invariant, because $V^{2}=12=0 \bmod 6$. At first sight the reverse, any orbifold shift $V_{\text {orb }}$, classified in Table 1 corresponds to a blow-up, does not seem to be 
true. However, we should take into account that two orbifold shift vectors are equivalent, i.e. lead to the same model, if: i) they differ by $3 \Lambda$ where $\Lambda$ is any element of the root lattice of $\mathrm{E}_{8} \times \mathrm{E}_{8}^{\prime}$, ii) they differ by sign flips of an even number of entries, or iii) are related by Weyl reflections. By suitable combining these operations one can show that all blow-up vectors of Table 2 can be obtained from the orbifold shifts in Table 1. (Only the first model in Table 1, characterized by the zero vector $\left(0^{8}\right)\left(0^{8}\right)$, does not have a blow-up counterpart in Table 2.) This leads to a direct matching between orbifold and blow-up models. Using the notation from the Table 1 and 2, we match model B with BI, model D with DI. We also see that even though CI and CII are different blow-up models, they correspond to the same orbifold theory C. The same applies to the $\mathrm{U}(1)$ bundle model AI and the standard embedding model AS (introduced in Section 2.2): They are both related to orbifold model $\mathrm{A}$.

Given the matching at the level of the gauge bundles, we can pass to checks at the level of the 4D gauge groups. A quick glance over the Table 1 and 2 shows that their gauge groups are never the same. This is easily explained from the orbifold perspective: the blow-up is generated by a non-vanishing vev of some twisted state, the so-called blow-up mode [25]. As all twisted states are charged, this vev induces a Higgs mechanism accompanied with gauge symmetry breaking and mass terms. It is not difficult to see from these tables that all non-Abelian blow-up gauge groups can be obtained from the orbifold gauge groups by switching on suitable vevs of twisted states.

Even after taking symmetry breaking, i.e. the branching of the representations of the orbifold state, into account the spectra of the orbifold models still do not agree with the ones of the resolved models: singlets w.r.t. non-Abelian blow-up groups, and some vector-like states are missing. Moreover, the U(1) charges of localized states do not coincide with the ones expected from the branchings. This can be confirmed from Table 3, for each model we give the orbifold spectrum (second column) and the resolution spectrum (third column).

All these differences can be understood by more carefully taking into account the possible consequences of a twisted state's vev $v$. After branching, this field is a singlet of the non-Abelian gauge group. In the quantum theory this means that the corresponding chiral superfield $\Psi_{q}$ with charge $q$ under the broken $U(1)$ never vanishes. Hence, it can be redefined as $\Psi_{q}=v e^{T}$, where $T$ is a new chiral superfield taking unconstraint values. As it transforms as an axion

$$
T \longrightarrow T+i q \phi
$$

under a $\mathrm{U}(1)$ transformation with parameter $\phi$, it is neutral. Hence, it is not part of the charged chiral spectrum computed using the Dirac index (14) on the resolution. In addition, we can use this axion chiral superfield $T$ to redefine the charges of other twisted states (see the last column of Table 3) so that all $U(1)$ charges of the twisted states agree with the ones of the localized resolution fields. For models CII and DI one needs in addition to change the U(1) basis when identifying the orbifold and blow-up states, if one enforces that the field getting a vev is only charged under the first blow-up U(1) factor. 


\begin{tabular}{|c|c|c|c|}
\hline & orbifold model & resolution model & field redefinitions \\
\hline \multirow[b]{2}{*}{\begin{tabular}{c||} 
A \\
$\downarrow$ \\
$\downarrow$ \\
AI
\end{tabular}} & $\mathrm{E}_{6} \times \mathrm{SU}(3) \times \mathrm{E}_{8}^{\prime}$ & $\mathrm{SO}(10) \times \mathrm{U}(3) \times \mathrm{E}_{8}^{\prime}$ & \multirow{2}{*}{$\begin{array}{l}(\mathbf{2 7}, \mathbf{1} ; \mathbf{1}) \rightarrow\left\{\begin{array}{l}(\mathbf{1}, \mathbf{1} ; \mathbf{1})_{-4}=e^{T} v \\
(\mathbf{1 6}, \mathbf{1} ; \mathbf{1})_{-1}=e^{T}(\mathbf{1 6}, \mathbf{1} ; \mathbf{1})_{3} \\
(\mathbf{1 0}, \mathbf{1} ; \mathbf{1})_{2}=e^{-T / 2}(\mathbf{1 0}, \mathbf{1} ; \mathbf{1})_{0}^{m}\end{array}\right. \\
(\mathbf{1}, \overline{\mathbf{3}} ; \mathbf{1}) \rightarrow(\mathbf{1}, \overline{\mathbf{3}} ; \mathbf{1})_{0}=e^{T}(\mathbf{1}, \overline{\mathbf{3}} ; \mathbf{1})_{4}\end{array}$} \\
\hline & $\begin{array}{r}\frac{1}{9}(\mathbf{2 7}, \mathbf{3} ; \mathbf{1}) \\
+(\mathbf{2 7}, \mathbf{1} ; \mathbf{1})+3(\mathbf{1}, \overline{\mathbf{3}} ; \mathbf{1}) \\
\end{array}$ & $\begin{array}{c}\frac{1}{9}\left[(\mathbf{1 6}, \mathbf{3} ; \mathbf{1})_{-1}+(\mathbf{1 0}, \mathbf{3} ; \mathbf{1})_{2}+(\mathbf{1}, \mathbf{3} ; \mathbf{1})_{-4}\right] \\
+(\mathbf{1 6}, \mathbf{1} ; \mathbf{1})_{3}+3(\mathbf{1}, \overline{\mathbf{3}} ; \mathbf{1})_{4}\end{array}$ & \\
\hline \multirow[b]{2}{*}{\begin{tabular}{c||}
$\mathrm{B}$ \\
$\downarrow$ \\
$\mathrm{BI}$ \\
\end{tabular}} & {$\left[\mathrm{E}_{6} \times \mathrm{SU}(3)\right]^{2}$} & {$\left[\mathrm{E}_{6} \times \mathrm{U}(2)\right]^{2}$} & \multirow[b]{2}{*}{$(\mathbf{1}, \mathbf{3} ; \mathbf{1}, \mathbf{3}) \rightarrow\left\{\begin{array}{l}(\mathbf{1}, \mathbf{2} ; \mathbf{1}, \mathbf{1})_{-1,3}=e^{T}(\mathbf{1}, \mathbf{2} ; \mathbf{1}, \mathbf{1})_{3,3} \\
(\mathbf{1}, \mathbf{1} ; \mathbf{1}, \mathbf{2})_{-1,-3}=e^{T}(\mathbf{1}, \mathbf{1} ; \mathbf{1}, \mathbf{2})_{3,-3} \\
(\mathbf{1}, \mathbf{2} ; \mathbf{1}, \mathbf{2})_{2,0}=e^{-T / 2}(\mathbf{1}, \mathbf{2} ; \mathbf{1}, \mathbf{2})_{0,0}^{m}\end{array}\right.$} \\
\hline & $\begin{array}{l}\frac{1}{9}[(\mathbf{2 7}, \overline{\mathbf{3}} ; \mathbf{1}, \mathbf{1}) \\
+(\mathbf{1}, \mathbf{1} ; \mathbf{2 7}, \overline{\mathbf{3}})] \\
\quad+(\mathbf{1}, \mathbf{3} ; \mathbf{1}, \mathbf{3}) \\
\end{array}$ & $\begin{array}{l}\frac{1}{9}\left[(\mathbf{2 7}, \mathbf{2} ; \mathbf{1}, \mathbf{1})_{-1,-1}+(\mathbf{2 7}, \mathbf{1} ; \mathbf{1}, \mathbf{1})_{2,2}\right. \\
\left.+(\mathbf{1}, \mathbf{1} ; \mathbf{2 7}, \mathbf{2})_{-1,1}+(\mathbf{1}, \mathbf{1} ; \mathbf{2 7}, \mathbf{1})_{2,-2}\right] \\
\quad+(\mathbf{1}, \mathbf{2} ; \mathbf{1}, \mathbf{1})_{3,3}+(\mathbf{1}, \mathbf{1} ; \mathbf{1}, \mathbf{2})_{3,-3} \\
\end{array}$ & \\
\hline \multirow[b]{2}{*}{\begin{tabular}{c||}
$\mathrm{C}$ \\
$\downarrow$ \\
$\mathrm{CI}$ \\
\end{tabular}} & $\mathrm{E}_{7} \times \mathrm{SO}(14) \times \mathrm{U}(1)^{2}$ & $\mathrm{E}_{7} \times \mathrm{SO}(14) \times \mathrm{U}(1)^{2}$ & \multirow{2}{*}{$\begin{array}{l}(\mathbf{1} ; \mathbf{1})_{-4,0}=e^{T} v \\
(\mathbf{1} ; \mathbf{1})_{0,4}=e^{T}(\mathbf{1} ; \mathbf{1})_{4,4} \\
(\mathbf{1} ; \mathbf{1 4})_{2,0}=e^{-T / 2}(\mathbf{1} ; \mathbf{1 4})_{0,0}^{m}\end{array}$} \\
\hline & $\begin{array}{c}\frac{1}{9}\left[(\mathbf{5 6} ; \mathbf{1})_{2,2}+(\mathbf{1} ; \mathbf{1})_{-4,-4}\right. \\
\left.+(\mathbf{1} ; \mathbf{6 4})_{-1,2}+(\mathbf{1} ; \mathbf{1 4})_{2,-4}\right]+ \\
(\mathbf{1} ; \mathbf{1 4})_{2,0}+(\mathbf{1} ; \mathbf{1})_{-4,0}+3(\mathbf{1} ; \mathbf{1})_{0,4} \\
\end{array}$ & $\begin{array}{c}\frac{1}{9}\left[(\mathbf{5 6} ; \mathbf{1})_{2,2}+(\mathbf{1} ; \mathbf{1})_{-4,-4}\right. \\
\left.+(\mathbf{1} ; \mathbf{6 4})_{-1,2}+(\mathbf{1} ; \mathbf{1 4})_{2,-4}\right] \\
+3(\mathbf{1} ; \mathbf{1})_{4,4} \\
\end{array}$ & \\
\hline \multirow[b]{2}{*}{$\begin{array}{c}\text { C } \\
\downarrow \\
\text { CII }\end{array}$} & $\mathrm{E}_{7} \times \mathrm{SO}(14) \times \mathrm{U}(1)^{2}$ & $\mathrm{E}_{7} \times \mathrm{SO}(12) \times \mathrm{U}(1)^{3}$ & \multirow{2}{*}{$\begin{array}{l}(\mathbf{1} ; \mathbf{1 4})_{2,0} \rightarrow\left\{\begin{array}{l}(\mathbf{1} ; \mathbf{1})_{-4,0,0}=e^{T} v \\
(\mathbf{1} ; \mathbf{1 2})_{-1,0,-2}=e^{T}(\mathbf{1} ; \mathbf{1 2})_{3,0,-2} \\
(\mathbf{1} ; \mathbf{1})_{2,0,-4}=e^{-T / 2}(\mathbf{1} ; \mathbf{1})_{0,0,-4}^{m}\end{array}\right. \\
(\mathbf{1} ; \mathbf{1})_{-4,0} \rightarrow(\mathbf{1} ; \mathbf{1})_{2,0,4}=e^{-T / 2}(\mathbf{1} ; \mathbf{1})_{0,0,4}^{m} \\
(\mathbf{1} ; \mathbf{1})_{0,4} \rightarrow(\mathbf{1} ; \mathbf{1})_{0,4,0}=e^{T}(\mathbf{1} ; \mathbf{1})_{4,4,0}\end{array}$} \\
\hline & $\begin{array}{c}\frac{1}{9}\left[(\mathbf{5 6} ; \mathbf{1})_{2,2}+(\mathbf{1} ; \mathbf{6 4})_{-1,2}\right. \\
\left.+(\mathbf{1} ; \mathbf{1})_{-4,-4}+(\mathbf{1} ; \mathbf{1 4})_{2,-4}\right]+ \\
(\mathbf{1} ; \mathbf{1 4})_{2,0}+(\mathbf{1} ; \mathbf{1})_{-4,0}+3(\mathbf{1} ; \mathbf{1})_{0,4}\end{array}$ & $\begin{array}{c}\frac{1}{9}\left[(\mathbf{5 6} ; \mathbf{1})_{-1,2,-2}+(\mathbf{1} ; \mathbf{3 2})_{-1,2,2}+(\mathbf{1} ; \overline{\mathbf{3 2}})_{2,2,0}\right. \\
\left.+(\mathbf{1} ; \mathbf{1})_{-4,-4,0}+(\mathbf{1} ; \mathbf{1 2})_{-1,-4,-2}+(\mathbf{1} ; \mathbf{1})_{2,-4, \pm 4}\right]+ \\
\quad(\mathbf{1} ; \mathbf{1 2})_{3,0,-2}+3(\mathbf{1} ; \mathbf{1})_{4,4,0}\end{array}$ & \\
\hline \multirow[b]{2}{*}{\begin{tabular}{c||} 
D \\
$\downarrow$ \\
DI
\end{tabular}} & $\mathrm{SU}(9) \times \mathrm{SO}(14) \times \mathrm{U}(1)$ & $\mathrm{SU}(8) \times \mathrm{SO}(14) \times \mathrm{U}(1)^{2}$ & \multirow[b]{2}{*}{$(\overline{\mathbf{9}} ; \mathbf{1})_{-4 / 3} \rightarrow\left\{\begin{array}{l}(\mathbf{1} ; \mathbf{1})_{-4,0}=e^{T} v \\
(\overline{\mathbf{8}} ; \mathbf{1})_{-1,3}=e^{T}(\overline{\mathbf{8}} ; \mathbf{1})_{3,3}\end{array}\right.$} \\
\hline & $\begin{array}{c}\frac{1}{9}\left[(\mathbf{8 4} ; \mathbf{1})_{0}+(\mathbf{1} ; \mathbf{6 4})_{-1}+(\mathbf{1} ; \mathbf{1 4})_{2}\right] \\
+(\overline{\mathbf{9}} ; \mathbf{1})_{-4 / 3}\end{array}$ & $\begin{array}{c}\frac{1}{9}\left[(\mathbf{5 6} ; \mathbf{1})_{-1,-1}+(\mathbf{2 8} ; \mathbf{1})_{2,2}+(\mathbf{1} ; \mathbf{6 4})_{-1,2}\right. \\
\left.\quad+(\mathbf{1} ; \mathbf{1 4})_{2,-4}\right]+(\overline{\mathbf{8}} ; \mathbf{1})_{3,3}\end{array}$ & \\
\hline
\end{tabular}

Table 3: We define the matching orbifold and blow-up models in the first column. The second and third columns give their orbifold and resolution spectra, respectively. The final column gives the field redefinitions necessary to match the two spectra. For blow-up models $\mathrm{CII}$ and DI a change of U(1) basis accompanies the branching (indicated by $\rightarrow$ ) to ensure that the state getting the vev is charged under the first blow-up U(1) only. The superscript $m$ indicates non-chiral states that get a mass in blow-up, and therefore decouple from the massless spectrum. 
Finally, the remaining states that are missing in blow-up (referred to in Table 3 with a superscript $m$ ) have Yukawa couplings with the blow-up mode, so that they get a mass term in the blow-up. Taking all these blow-up effects into account shows that the spectra of the blown-up orbifold and resolution models become perfectly identical.

\subsection{F- and D- flatness of the blow-up mode}

In the matching of heterotic orbifold models with their resolved counterparts we assumed that a single twisted field of the orbifold model was responsible for generating the blowup. No other twisted or untwisted states attained non-vanishing vevs. However, in order to obtain a supersymmetric configuration, we have to pay attention to possible nonvanishing $\mathrm{F}$-terms arising from the non-zero vev. The analysis of the $\mathrm{F}$-flatness for a superpotential $\mathcal{W}$ is rather involved in the context of heterotic orbifold model building, because in principle it contains an infinite set of terms with coefficients determined by complicated string amplitudes. In practice string selection rules can be used to argue that a large class of these coefficients vanishes identically, while the others are taken to be arbitrary [26, 27, 28].

Our assumption above that only a single twisted superfield has a non-vanishing vev greatly simplifies the F-flatness analysis: non-vanishing F-terms can only arise from terms in the superpotential that are at most linear in fields having zero vevs. As in most of the cases the vanishing vev fields form non-Abelian representations gauge invariance of the superpotential implies that they cannot appear linearly. This means that the complicated analysis of the superpotential involving many superfields often reduces to the analysis of a complex function of a single variable. In what follows we show that all the blow-ups described previously are F-flat and therefore constitute viable resolutions of orbifold models.

Non-vanishing D-terms can only arise under the following conditions [29]: let $\varphi_{q}$ be the scalar component of the only superfield $\Psi_{q}$ that acquires a vev $\left\langle\varphi_{q}\right\rangle$ as discussed above. The D-terms are proportional to $D^{a} \sim \bar{\varphi}_{q} T^{a} \varphi_{q}$, where $T_{a}$ are the generators of the orbifold gauge group $G$. Therefore, certainly all D-terms corresponding to the generators $T_{a}$ that annihilate $\left\langle\varphi_{q}\right\rangle$ vanish. They generate the little group $H$ of gauge symmetries unbroken by the vev. Consequently, non-vanishing D-terms are only possible for the generators $T^{a}$ of the coset $G / H$. Under an infinitesimal gauge transformation with parameter $\epsilon$ the D-terms transform as $D^{a} \rightarrow D^{a}+\bar{\varphi}_{q}\left[\epsilon, T^{a}\right] \varphi_{q}$. This means that for all generators $T^{a}$ which do not commute with all generators of $G / H$, we can find a gauge such that the $D^{a}$ 's associated to them vanish. But since $\left(D^{a}\right)^{2}$ defines a gauge invariant object, all these $D^{a}$ have to vanish in any gauge. The only possibly non-vanishing Dterms correspond to the Abelian subgroup of the coset $G / H$. As we explain in the next subsection, precisely those D-terms, which are associated with anomalous U(1)'s on the resolution $\mathcal{M}^{3}$, are non-vanishing. Apart from this subtle issue, D-flatness is automatically guaranteed. 
Matching $\mathrm{A} \rightarrow \mathrm{AS}$ by a vev of $3_{H}(1, \overline{3} ; 1)$

We begin our analysis with the standard embedding defined by a gauge bundle with $i \mathcal{F}=\mathcal{R}$. Because both transform by conjugation, $i \mathcal{F} \rightarrow g^{-1} i \mathcal{F} g$ and $\mathcal{R} \rightarrow g_{H}^{-1} \mathcal{R} g_{H}$ under gauge and internal local Lorentz (holonomy) transformations, respectively, we know that these gauge transformations are identified: $g=g_{H}$. This fact will help us to identify the blow-up field in the following.

To reconstruct the corresponding blow-up mode we need a field that transforms under both of these transformations. In orbifold model A the only candidates for this are the three triplets $3(\mathbf{1}, \overline{\mathbf{3}} ; \mathbf{1})$. The multiplicity three is due to internal oscillator excitations of these states, i.e. these states form a triplet under the R-symmetry group $\mathrm{SU}(3)_{R}$ as it commutes with the $\mathbb{Z}_{3}$ orbifold holonomy, which in turn is proportional to the identity. A more precise way of referring to these states is therefore: $\mathbf{3}_{R}(\mathbf{1}, \overline{\mathbf{3}} ; \mathbf{1})$; we can view them collectively as a $3 \times 3$ matrix $G^{\alpha}{ }_{i}$, where $\alpha$ is the $\mathrm{SU}(3)_{R}$ index and $i$ the $\mathrm{SU}(3)$ index 3 . The $\mathrm{SU}(3)$ gauge and $\mathrm{SU}(3)_{R} \mathrm{R}$-symmetry groups act on it as $G \rightarrow g_{R} G g^{-1}$. Since any complex matrix $M$ can be written as a product $M=U H$ of a unitary matrix $U$ and a Hermitian matrix $H$, which in turn can be diagonalized by another unitary matrix $V$ as $H=V D V^{-1}$ with $D$ a real diagonal matrix, we can use the gauge and $\mathrm{R}$-symmetry transformations to bring $G$ in a real diagonal form. If we choose the vev matrix $G$ to be proportional to the identity: $G=v \mathbf{1}$, we find that only a diagonal gauge and $\mathrm{R}$-symmetry transformation preserves this vev. This means that in the blow-up the symmetry $\mathrm{SU}(3)_{R} \times \mathrm{SU}(3)$ is broken to the diagonal $\mathrm{SU}(3)$ subgroup (with $g=g_{R}$ ). Comparing to the standard embedding on the resolution (with $g=g_{H}$ ), the vev of $G$ changed the $\mathbb{Z}_{3}$ orbifold holonomy to the full SU(3) holonomy of a Calabi-Yau.

To understand whether such a vev for $G$ is possible, we need to analyze the superpotential of the theory. Because the $\mathbb{Z}_{3}$ action is proportional to the identity, the $\mathbb{C}^{3} / \mathbb{Z}_{3}$ orbifold theory is left invariant by any unitary transformation $\mathrm{U}(3)_{R}$ of the internal coordinates, there is no superpotential involving only $G$ : the $\mathrm{SU}(3) \times \mathrm{SU}(3)_{R}$ invariance requires such superpotential to be a function of $\operatorname{det} G$, but that is not invariant under $\mathrm{U}(1)_{R}$. (Similar arguments for the compact $T^{6} / \mathbb{Z}_{3}$ allow a cubic superpotential for $G$ in that case [16].) Hence, any vev for $G$ defines a F-flat direction. However, it can be seen that D-flatness requires it to be of the form above [16]: The SU(3) D-terms correspond to the traceless part of the matrix $D^{i}{ }_{j}=\bar{G}^{i}{ }_{\alpha} G^{\alpha}{ }_{j}$. In the diagonal form the matrix $G$ has the vevs $v_{1}, v_{2}$ and $v_{3}$ as its diagonal elements. This means that $D$ is a diagonal matrix with entries $v_{1}^{2}, v_{2}^{2}$ and $v_{3}^{2}$. But this has a non-vanishing traceless part unless all vevs are equal.

\section{Matching $\mathrm{A} \rightarrow \mathrm{AI}$ by a vev of $(27,1 ; 1)$}

In this case the blow-up mode is in the twisted state $(\mathbf{2 7}, \mathbf{1} ; \mathbf{1})$, and the relevant part of the superpotential are formed from its cubic $\mathrm{E}_{6}$ invariant

$$
\mathcal{W} \sim[(27,1 ; 1)]^{3}+\ldots
$$

\footnotetext{
${ }^{3}$ We use the subscript notation to indicate that it is in the complex conjugate representation.
} 
Here, the notation $\sim$ indicates that we only give the lowest order gauge invariant structure ignoring its (order one) coefficient, and $+\ldots$ means that there is a whole power series of this invariant, restricted by some string selection rules. Such a superpotential is always $\mathrm{F}$-flat. To see this, consider the branching of the twisted state $\mathbf{2 7} \rightarrow \mathbf{1 6}_{-1}+\mathbf{1 0}_{2}+\mathbf{1}_{-4}$ due to its own vev $v$ that breaks $\mathrm{E}_{6} \rightarrow \mathrm{SO}(10) \times \mathrm{U}(1)$. In terms of $\mathrm{SO}(10) \times \mathrm{U}(1)-$ representations the invariant reads as

$$
[27]^{3}=16_{-1} \times 16_{-1} \times 10_{2}+10_{2} \times 10_{2} \times 1_{-4} .
$$

Since the $\mathbf{1}_{-4}$ represents the blow-up mode, and hence by definition the $\mathbf{1 6}_{-1}$ and $\mathbf{1 0}_{2}$ have zero vev, $\mathrm{F}$-flatness is automatically guaranteed. It is clear from this decomposition of the cubic invariant that the $\mathbf{1 0}_{2}$ becomes massive and decouples, while the $\mathbf{1 6}_{-1}$ stays strictly massless. This is in agreement with the blow-up spectrum given in Table 3 .

\section{Matching $\mathrm{B} \rightarrow \mathrm{BI}$ by a vev of $(1,3 ; 1,3)$}

In orbifold model B the only twisted state is a $(\mathbf{1}, \mathbf{3} ; \mathbf{1}, \mathbf{3})$-plet, hence it is the only possible blow-up mode. Like the blow-up mode in the case of standard embedding AS discussed above, this blow-up mode defines a $3 \times 3$ matrix denoted by $C$. Gauge transformations with $g \in \mathrm{SU}(3)$ and $g^{\prime} \in \mathrm{SU}(3)^{\prime}$ act via left and right multiplication $C \rightarrow g C g^{\prime}$. The relevant part of the superpotential is therefore also very similar

$$
\mathcal{W} \sim \operatorname{det} C+\ldots
$$

As the two $\mathrm{SU}(3)$ 's are independent, we can again assume that the matrix $C$ is diagonalized. To obtain the appropriate symmetry breaking $\mathrm{SU}(3) \rightarrow U(2)$ of both $\mathrm{SU}(3)$ s, only one of the three diagonal elements has a non-vanishing vev. This is a very different orientation of the vev as compared to the standard embedding. Expanding the superpotential around this vev, shows that the state $(\mathbf{1}, \mathbf{2} ; \mathbf{1}, \mathbf{2})_{0,0}^{m}$ becomes massive.

\section{Matching $\mathrm{C} \rightarrow \mathrm{CI}$ by a vev of $(\mathbf{1}, \mathbf{1})_{-4,0}$}

In orbifold model $\mathrm{C}$ we can construct gauge invariant structures for the superpotential only by combining the states $(\mathbf{1}, \mathbf{1})_{-4,0}$, and $(\mathbf{1}, \mathbf{1 4})_{2,0}$

$$
\mathcal{W} \sim(\mathbf{1}, \mathbf{1})_{-4,0}\left[(\mathbf{1}, \mathbf{1 4})_{2,0}\right]^{2}+\ldots
$$

Since the CI blow-up is realized by giving a vev to the orbifold state $(\mathbf{1}, \mathbf{1})_{-4,0}$, which is always coupled to pairs of $(\mathbf{1}, \mathbf{1 4})_{2,0}$ 's in the superpotential, this vev defines a flat direction of the potential and a mass term for the $(\mathbf{1}, \mathbf{1 4})_{2,0}$ is generated, provided that we perform the field redefinition indicated in Table 3. Hence, this state decouples.

\section{Matching $\mathrm{C} \rightarrow \mathrm{CII}$ by a vev of $(\mathbf{1}, \mathbf{1 4})_{2,0}$}

The CII blow-up is obtained when $(\mathbf{1}, \mathbf{1 4})_{2,0}$ gets a vev. Naively one expects that a vev for this state would lead to a symmetry breaking $\mathrm{SO}(14) \rightarrow \mathrm{SO}(13)$, but this is not 
in agreement with Table 3. To understand what is happening we have to consider the possible orientations of such a vev $C_{m}$, where $m$ denotes the $\mathrm{SO}(14)$ vector index. Since all states are chiral multiplets, we cannot use the real group $\mathrm{SO}(14)$ to put the vev in a single component. Indeed, writing $C_{m}=R_{m}+i J_{m}$ where $R_{m}$ and $J_{m}$ are real, we see that one can use a $\mathrm{SO}(14)$ transformation to obtain $R=\left(r, 0^{13}\right)$. This orientation is left invariant by $\mathrm{SO}(13)$ subgroup. This subgroup can be used to bring $J$ to the form $J=\left(j_{1}, j_{2}, 0^{12}\right)$. Hence, for generic values of $r, j_{1}$ and $j_{2}$ only the $\mathrm{SO}(12)$ subgroup is left unbroken, as Table 3 implies. Furthermore, the superpotential contains again the coupling (20). To have the auxiliary component of the superfield $(\mathbf{1}, \mathbf{1})_{-4,0}$ vanishing in extremum, the vev of the $\mathrm{SO}(14)$ invariant

$$
C^{T} C=r^{2}-j_{1}^{2}-j_{2}^{2}+2 i r j_{1}
$$

has to vanish. The only non-vanishing solution has: $j_{1}=0$ and $j_{2}^{2}=r^{2}=v^{2}$. This vev induces a mass by pairing up one of the singlets from the branching of $14 \rightarrow \mathbf{1 2}+\mathbf{1}+\mathbf{1}$ with the singlet already present in the orbifold spectrum, see Table 3 .

Notice that there is a third field in model $C$ that could have a non-zero vev, the R-symmetry triplet $\mathbf{3}_{R}(\mathbf{1} ; \mathbf{1})_{0,4}$. This superfield cannot appear in any superpotential by itself, this means that any vev for this superfield leads to a supersymmetric configuration. Nevertheless, we do not have any candidate for a U(1) gauge configuration on the resolution that corresponds to this vev.

\section{Matching $\mathrm{D} \rightarrow \mathrm{DI}$ by a vev of $(\overline{\mathbf{9}}, 1)_{-4 / 3}$}

Finally, we consider the orbifold model D. As it has only one charged twisted state $(\overline{\mathbf{9}}, 1)_{-4 / 3}$ it is not possible, due to the string selection rules, to write down any superpotential with terms at most linear in the other fields. Thus, it can attain any vev leading to the symmetry breaking as described in Table 3 ,

\section{Other gauge bundles on the resolution?}

The list of possible vevs of twisted states of a given heterotic orbifold model is exhausted only for the last case, model D. The other models allow other blow-ups in principle:

First of all, model $C$ also has an $\mathrm{SU}(3)_{R}$ triplet of scalars, there is no obvious reason why one of them cannot have a non-vanishing vev. Model B allows for other possible orientations for the vev of the $(\mathbf{1}, \mathbf{3} ; \mathbf{1}, \mathbf{3})$ state, because it defines a $3 \times 3$-matrix with three eigenvalues. Thus, in general one should allow for blow-ups defined by a multitude of vevs for possibly all the twisted states that a given orbifold model possesses.

Since our classification of Abelian gauge bundle models on the resolution of $\mathbb{C}^{3} / \mathbb{Z}_{3}$ is complete, and we have identified the blow-up modes in the various heterotic theories leading to these models, we conclude that other (multiple field) vevs correspond to nonAbelian bundles on the resolution. Aside from the standard embedding, model AS, their classification is beyond the scope of this paper. 


\subsection{Multiple anomalous U(1)'s on the blow-up}

In this section we investigate the anomaly cancellation and D-flatness on the resolution $\mathcal{M}^{3}$. We find that there can be at most two anomalous $\mathrm{U}(1)$ 's, and that their cancellation involves two axions [30, 31, the model-independent and a model-dependent one 4 We show that the counterpart of such an anomaly cancellation, from the orbifold perspective, is a mixture of the standard orbifold Green-Schwarz mechanism and the Higgs mechanism related to the blow-up mode. From this we deduce relations between the two axions and their orbifold counterparts, namely, the universal axion of heterotic orbifold models and a second field related to the blow-up mode. Finally we discuss the issue of D-flatness of the resolution. We show that the blow-up is not along a D-flat direction. Rather, in the blow-up a constant D-term is produced, which is matched, from the resolution perspective, with the appearance of a new Fayet-Iliopoulos term due to the presence of two, rather then one anomalous $\mathrm{U}(1)$ 's.

\section{Anomalous U(1)'s on the resolution: the axions}

We deduce the $4 \mathrm{D}$ anomaly polynomial $\hat{I}_{6}$ from dimensional reduction of the $10 \mathrm{D}$ one, $\hat{I}_{12}$. For notational convenience we absorb some factors $2 \pi i$ in the definition of the anomaly polynomial: $\hat{I}_{2 n+2}=(2 \pi i)^{n} I_{2 n+2}$. The anomaly polynomial factorizes as $\hat{I}_{12}=X_{4} \cdot X_{8}$, where 33, 34]

$$
\begin{gathered}
X_{4}=\operatorname{tr} \mathfrak{R}^{2}-\operatorname{tr}(i \mathfrak{F})^{2} \\
X_{8}=\frac{1}{96}\left[\frac{\operatorname{Tr}(i \mathfrak{F})^{4}}{24}-\frac{\left(\operatorname{Tr}(i \mathfrak{F})^{2}\right)^{2}}{7200}-\frac{\operatorname{Tr}(i \mathfrak{F})^{2} \operatorname{tr} \mathfrak{R}^{2}}{240}+\frac{\operatorname{tr} \Re^{4}}{8}+\frac{\left(\operatorname{tr} \Re^{2}\right)^{2}}{32}\right],
\end{gathered}
$$

with $\mathfrak{R}$ denoting the $10 \mathrm{D}$ curvature. The trace $\operatorname{tr}$ in the "fundamental" of $\mathrm{E}_{8} \times \mathrm{E}_{8}^{\prime}$ is formally defined via $\operatorname{tr}=\frac{1}{30} \mathrm{Tr}$, Tr being the standard trace in the adjoint representation. From $\hat{I}_{12}$ the $4 \mathrm{D}$ anomaly polynomial $\hat{I}_{6}$ can be derived via an integration over the resolution manifold. The integration will be performed after inserting the expansions $\mathfrak{R}=R+\mathcal{R}$ and $i \mathfrak{F}=i F+i \mathcal{F}_{V}$ and splitting the forms $X_{4}$ and $X_{8}$ according to

$$
\hat{I}_{12}=X_{4,0} X_{2,6}+X_{2,2} X_{4,4}+X_{0,4} X_{6,2}
$$

where we read $X_{a, b}$ as an $(a+b)$-form with $a$ indices in the $6 \mathrm{D}$ internal and $b$ indices in the $4 \mathrm{D}$ Minkowski space. Since the backgrounds are such that the $H_{3}$ Bianchi Identity is fulfilled, the $4 \mathrm{D}$ anomaly polynomial $\hat{I}_{6}$ is written as the factorized sum

$$
\hat{I}_{6} \equiv \frac{1}{(2 \pi i)^{3}} \int_{\mathcal{M}^{3}} \hat{I}_{12}=\frac{1}{(2 \pi i)^{3}} \int_{\mathcal{M}^{3}}\left(X_{2,2} X_{4,4}+X_{0,4} X_{6,2}\right) \text {. }
$$

Inserting the expressions for $X_{2,2}$ and $X_{0,4}$ in terms of the field strengths and rearranging the terms on the right hand side yields

$$
\hat{I}_{6}=\hat{I}_{6}^{u n i}+\hat{I}_{6}^{n o n} \quad \text { with } \quad \hat{I}_{6}^{u n i}=X_{2}^{u n i} \cdot X_{0,4} \quad \text { and } \quad \hat{I}_{6}^{n o n}=X_{2}^{n o n} \cdot X_{4}^{n o n}
$$

\footnotetext{
${ }^{4}$ For a recent review on axions from string theory see 32 .
} 
where $X_{2}^{\text {non }}=-2 \operatorname{tr}\left[H_{V} i F\right], X_{0,4}=\operatorname{tr} R^{2}-\operatorname{tr}(i F)^{2}$, and

$$
X_{2}^{\text {uni }}=\frac{1}{(2 \pi i)^{3}} \int_{\mathcal{M}^{3}} X_{6,2}, \quad X_{4}^{n o n}=\frac{1}{(2 \pi i)^{3}} \int_{\mathcal{M}^{3}} i \mathcal{F} X_{4,4} .
$$

Now the integration is performed and results in

$$
\begin{gathered}
X_{2}^{u n i}=-\frac{1}{96} \operatorname{Tr}\left[\left(\frac{1}{18} H_{V}^{3}-\frac{1}{5} H_{V}\right)(i F)\right], \\
X_{4}^{n o n}=-\frac{1}{192}\left[\operatorname{Tr}\left[\left(\frac{1}{6} H_{V}^{2}-\frac{1}{5}\right)(i F)^{2}\right]-\frac{1}{3 \cdot 30^{2}}\left(\operatorname{Tr}\left[H_{V}(i F)\right]\right)^{2}-\operatorname{tr} R^{2}\right] .
\end{gathered}
$$

These equations describe how the $4 \mathrm{D}$ anomaly $\hat{I}_{6}$ can be written as a sum of two factorized parts, a universal part $\hat{I}_{6}^{u n i}$ and a non-universal part $\hat{I}_{6}^{\text {non }}$. Since both parts are proportional to $\operatorname{tr}(i F)$, they are non-vanishing only for anomalous $\mathrm{U}(1)$-factors. As we started from an anomaly free theory in 10D the 4D Green-Schwarz mechanism will cancel the two summands in $\hat{I}_{6}$ by two axions. The universal anomaly is canceled by the anomalous variation of the model-independent axion and the non-universal part by the model-dependent axion, as shown in the following. Therefore on the resolution of $\mathbb{C}^{3} / \mathbb{Z}_{3}$ there can be at most two anomalous U(1)'s. In Table 4 , we give the anomaly terms for each of the $\mathbb{C}^{3} / \mathbb{Z}_{3}$ models.

The $4 \mathrm{D}$ anomaly must be canceled by the anomalous variation of the $10 \mathrm{D}$ two-form $B_{2}$, which can be expanded as

$$
B_{2}=b_{2}+i \mathcal{F} b_{0}+\omega_{2} B_{0}
$$

The Kähler form $\omega_{2}$, obtained from the Kähler potential, and the U(1) gauge bundle field strength $i \mathcal{F}$ are harmonic two-forms on the resolution $\mathcal{M}^{3}$. Thus $b_{0}$ and $B_{0}$ are $4 \mathrm{D}$ massless scalars. In addition, $b_{2}$ is a two-form in Minkowski space, the $4 \mathrm{D}$ B-field. The gauge transformation of the two-form $b_{2}$ and the scalar $b_{0}$ are determined by the expansion of the three-form field strength [17]

$$
H_{3}=d b_{2}+\Omega_{Y M}-\Omega_{L}+\omega_{2} d B_{0}+i \mathcal{F}\left(d b_{0}-2 \operatorname{tr}\left[H_{V} i A\right]\right),
$$

where $\Omega_{Y M}$ and $\Omega_{L}$ are the Yang-Mills and Lorentz Chern-Simons three forms, respectively. This implies that $B_{0}$ has no anomalous variations, only $b_{2}$ and $b_{0}$ can take part in the 4D Green-Schwarz mechanism. In particular, $b_{0}$ transforms under a gauge transformation as

$$
\delta_{\Lambda} b_{0}=-2 \operatorname{tr}\left[H_{V} \Lambda\right]
$$

where $\Lambda$ is the gauge parameter. Therefore, the scalar $b_{0}$ and the Poincaré-dual of $b_{2}$ can be interpreted as axions, since the anomaly cancellation on the resolution occurs via the usual coupling of the B-field [33]

$$
\frac{1}{(2 \pi i)^{3}} \int_{\mathbb{M}^{4} \times \mathcal{M}^{3}} B_{2} X_{8} \supset \frac{1}{(2 \pi i)^{3}} \int_{\mathbb{M}^{4} \times \mathcal{M}^{3}}\left(b_{2} X_{6,2}+i \mathcal{F} b_{0} X_{4,4}\right)=\int_{\mathbb{M}^{4}}\left(b_{2} X_{2}^{\text {uni }}+b_{0} X_{4}^{\text {non }}\right) \text {. }
$$




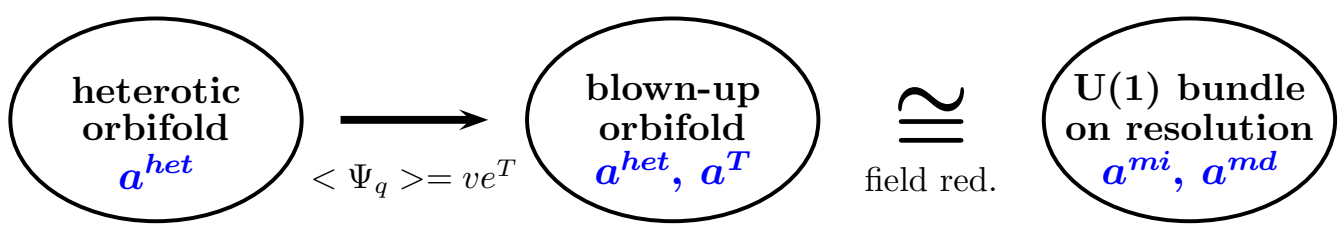

Figure 2: Schematic picture of the blowing-up procedure. A twisted field (blow-up mode) on the orbifold aquires a vev and its phase degree of freedom is reinterpreted as the axion $a^{T}$. As $\mathrm{U}(1)$-charges of some of the twisted fields are still different from the ones on the resolution $\mathcal{M}^{3}$, appropriate field-redefinitions are necessary to make them coincide.

The dual of $b_{2}$ is the model-independent axion $a^{m i}$, because its existence does not depend on the particular internal manifold. The scalar $b_{0}$ defines the model-dependent axion $a^{m d}$, i.e. $a^{m d}=b_{0}$. The model-dependent axion is a localized state, as the field strength $i \mathcal{F}$ becomes strongly peaked at the singularity in the orbifold limit. This means that it should be interpreted as a twisted state from the orbifold perspective.

\section{Relations between the various axions}

The matching of the spectra involves field redefinitions using a superfield $T$ associated to the blow-up mode. We showed that $T$ transforms with a shift under $\mathrm{U}(1)$-gauge transformations. This means that the imaginary part of $T$ transforms like an axion, which we denote as $a^{T}$. We now investigate whether $a^{T}$ can be interpreted as the modeldependent axion $a^{m d}$ of the corresponding resolution and to what extend the heterotic axion $a^{\text {het }}$ is related to the model-independent axion $a^{m i}$. A schematic picture of the context in which these different axions are defined is given in figure 2.

The field redefinitions are necessary to obtain the matching of the orbifold and blowup spectra implying a modification of the anomaly polynomial for the heterotic orbifold model. First of all, we have to take into account that the orbifold gauge group is broken in the blow-up. Thus, the anomaly polynomial should also be re-expressed in terms of the new unbroken gauge group factors. The anomaly polynomial $\hat{I}_{6}^{\text {het }}=X_{2}^{\text {het }} \cdot X_{0,4}$ describes the anomaly of the heterotic orbifold before the field redefinition but after the branching.

Moreover, the field redefinitions generate a new anomaly polynomial $\hat{I}_{6}^{r e d}=i q F X_{4}^{\text {red }}$. Since they modify the U(1) charges of twisted superfields $\hat{I}_{6}^{r e d}$ is proportional to the field strength $i F$ of this $\mathrm{U}(1)$. Thus, the anomaly polynomial of the blow-up equals the sum of $\hat{I}_{6}^{\text {het }}$ and the contribution from the anomalous field redefinition:

$$
\hat{I}_{6}^{\text {het }}+\hat{I}_{6}^{\text {red }}=\hat{I}_{6}^{\text {blow }}=\hat{I}_{6}^{u n i}+\hat{I}_{6}^{\text {non }} .
$$

In Table 4 we list $\hat{I}_{6}^{\text {het }}$, model by model, computed from the orbifold model spectra, and $\hat{I}_{6}^{u n i}$ and $\hat{I}_{6}^{\text {non }}$, computed as discussed above.

The anomaly cancellation on the orbifold after the field redefinitions involves the heterotic axion $a^{\text {het }}$ and the localized twisted axion $a^{T}$. Equation (34) implies the relation 


\begin{tabular}{|c|c|c|}
\hline & anomaly polynomials & $\alpha$ \\
\hline $\begin{array}{c}\text { A } \\
\downarrow \\
\text { AI }\end{array}$ & $\begin{array}{l}\hat{I}_{6}^{\text {het }}=0 \\
\hat{I}_{6}^{\text {uni }}=\frac{3}{2}(i F)\left[24(i F)^{2}+\left(i F_{10}\right)^{2}+2\left(i F_{3}\right)^{2}+\left(i F_{8}\right)^{2}-R^{2}\right] \\
\hat{I}_{6}^{\text {non }}=\frac{1}{4}(i F)\left[528(i F)^{2}+6\left(i F_{10}\right)^{2}+12\left(i F_{3}\right)^{2}-6\left(i F_{8}\right)^{2}-R^{2}\right]\end{array}$ & $-\frac{3}{8}$ \\
\hline $\begin{array}{c}\mathrm{B} \\
\downarrow \\
\mathrm{BI}\end{array}$ & $\begin{array}{l}\hat{I}_{6}^{\text {het }}=0 \\
\hat{I}_{6}^{\text {uni }}=0 \\
\hat{I}_{6}^{\text {non }}=\frac{1}{4}(i F)\left[96(i F)^{2}+288\left(i F^{\prime}\right)^{2}-R^{2}\right]\end{array}$ & 0 \\
\hline $\begin{array}{c}\mathrm{C} \\
\downarrow \\
\text { CI }\end{array}$ & $\begin{array}{l}\hat{I}_{6}^{\text {het }}=\frac{2}{3}\left(i F+i F^{\prime}\right)\left[24(i F)^{2}+48\left(i F^{\prime}\right)^{2}+\frac{1}{6}\left(i F_{7}\right)^{2}+\left(i F_{14}\right)^{2}-R^{2}\right] \\
\hat{I}_{6}^{\text {uni }}=\frac{1}{6}\left(i F+4 i F^{\prime}\right)\left[24(i F)^{2}+48\left(i F^{\prime}\right)^{2}+\frac{1}{6}\left(i F_{7}\right)^{2}+\left(i F_{14}\right)^{2}-R^{2}\right] \\
\hat{I}_{6}^{\text {non }}=\frac{1}{4}(i F)\left[144(i F)^{2}+480\left(i F^{\prime}\right)^{2}+384(i F)\left(i F^{\prime}\right)+\frac{1}{3}\left(i F_{7}\right)^{2}-2\left(i F_{14}\right)^{2}-R^{2}\right]\end{array}$ & $\frac{1}{8}$ \\
\hline $\begin{array}{c}\text { C } \\
\downarrow \\
\text { CII }\end{array}$ & $\begin{array}{l}\hat{I}_{6}^{h e t}=-\frac{1}{3}\left(i F-\frac{1}{2} i F^{\prime}+2 i F^{\prime \prime}\right) \times \\
\quad \times\left[24(i F)^{2}+3\left(i F^{\prime}\right)^{2}+32\left(i F^{\prime \prime}\right)^{2}+\frac{1}{6}\left(i F_{7}\right)^{2}+\left(i F_{12}\right)^{2}-R^{2}\right] \\
\hat{I}_{6}^{u n i}=\frac{1}{12}\left(17 i F+2 i F^{\prime}-8 i F^{\prime \prime}\right) \times \\
\quad \times\left[24(i F)^{2}+3\left(i F^{\prime}\right)^{2}+32\left(i F^{\prime \prime}\right)^{2}+\frac{1}{6}\left(i F_{7}\right)^{2}+\left(i F_{12}\right)^{2}-R^{2}\right] \\
\hat{I}_{6}^{\text {non }}=\frac{1}{4}(i F)\left[288(i F)^{2}+12\left(i F^{\prime}\right)^{2}+128\left(i F^{\prime \prime}\right)^{2}+96(i F)\left(i F^{\prime}\right)\right. \\
\left.\quad-384(i F)\left(i F^{\prime \prime}\right)-\frac{2}{3}\left(i F_{7}\right)^{2}+4\left(i F_{12}\right)^{2}-R^{2}\right]\end{array}$ & $-\frac{1}{4}$ \\
\hline $\begin{array}{l}\mathrm{D} \\
\downarrow \\
\mathrm{DI}\end{array}$ & $\begin{array}{l}\hat{I}_{6}^{h e t}=-\frac{1}{3}\left(i F-2 i F^{\prime}\right)\left[24(i F)^{2}+48\left(i F^{\prime}\right)^{2}+2\left(i F_{8}\right)^{2}+\left(i F_{14}\right)^{2}-R^{2}\right] \\
\hat{I}_{6}^{u n i}=\frac{1}{6}\left(i F+4 i F^{\prime}\right)\left[24(i F)^{2}+48\left(i F^{\prime}\right)^{2}+2\left(i F_{8}\right)^{2}+\left(i F_{14}\right)^{2}-R^{2}\right] \\
\hat{I}_{6}^{\text {non }}=\frac{1}{4}(i F)\left[192(i F)^{2}+480\left(i F^{\prime}\right)^{2}+384(i F)\left(i F^{\prime}\right)+4\left(i F_{8}\right)^{2}-2\left(i F_{14}\right)^{2}-R^{2}\right]\end{array}$ & $-\frac{1}{8}$ \\
\hline
\end{tabular}

Table 4: The anomalies of the blow-ups are compared with those of the orbifold theories. The resolution anomaly polynomial $\hat{I}_{6}^{\text {blow }}$ is divided into a universal part $\hat{I}_{6}^{\text {uni }}$ and a non-universal part $\hat{I}_{6}^{n o n}$. The axion redefinition parameter $\alpha$ is defined in Eq. (36). Note that we omitted the trace tr for the curvature and all non-abelian gauge group factors.

between the couplings of the various axions

$$
a^{\text {het }} X_{0,4}+a^{T} X_{4}^{\text {red }}=a^{m i} X_{0,4}+a^{m d} X_{4}^{\text {non }} .
$$

For a given model all the four-forms $X$ can be computed and (35) yields a system of linear equations for each group factor. This system can be solved and results in the following relations between the various axions

$$
a^{m i}=a^{h e t}+\alpha a^{T}, \quad a^{m d}=\beta a^{T},
$$

with $\alpha, \beta$ in general being model dependent constants. The normalization of the axions is chosen such that for all $\mathbb{C}^{3} / \mathbb{Z}_{3}$ models $\beta=-\frac{1}{16}$ by requiring that the blow-up modes always carry the same charge. Thus, only the coefficient $\alpha$ is model dependent and listed in the last column of Table 4. 


\subsection{D-terms in directions of anomalous $\mathrm{U}(1)$ 's}

Since there is always a single twisted chiral superfield getting a vev, there can be a nonvanishing D-term only for one broken gauge symmetry generator. Moreover, since such a field is just a singlet of the non-Abelian blow-up gauge group, there is a D-term only for a combination of the U(1)'s under which such a singlet $\Psi_{q}$ is charged. The presence of such a D-term is consistent: the non-vanishing D-term on the blown-up orbifold corresponds to an FI-term on the resolution. In spite of the original orbifold having at most a single anomalous $\mathrm{U}(1)$ and thus a single FI-term, the resolved models can have two. The second one is just the counterpart of the D-term generated by the vev. Hence, we conclude that D-flatness is guaranteed for all generators except the one corresponding to the broken $\mathrm{U}(1)$. But this non-vanishing $\mathrm{D}$-term is required to make the FI-terms coincide: on the level of local blow-ups, we match two dynamically unstable models.

Let us comment on how it is possible that a configuration chosen to be supersymmetric, i.e. which satisfies the Hermitian Yang-Mills equations (7), leads to non-vanishing D-terms. As was emphasized in [23], the Hermitian Yang-Mills equations get loop corrections precisely when anomalous $U(1)$ 's are present on smooth compactifications. In the analysis of this paper we have ignored such loop effects in the blow-up. The presence of non-vanishing D-terms for anomalous U(1)'s is simply signaling this.

We will see at the end of the next section, Section 3.6, that D-flatness can be ensured in the compact case. There, we will use the local models (with $D \neq 0$ ) as building blocks for the construction of compact ones and present various methods to obtain D-flatness afterwards.

\section{Blowing up the compact $T^{6} / \mathbb{Z}_{3}$ orbifold}

The local study of orbifold singularities captures a lot of the physics of compact orbifolds. The compact case has some important new aspects as we demonstrate by studying the blow-up of the $T^{6} / \mathbb{Z}_{3}$ orbifold. The latter is a space which is flat everywhere except at the 27 fixed points. For later use we enumerate the fixed points as $f=\left(f_{1}, f_{2}, f_{3}\right)$ with $f_{i}=0,1,2$. The fixed point $0=(0,0,0)$ is obviously localized at the origin. The index $i$ labels the three complex $T^{2}$ directions. The fixed points are singular and the singularity is identical to the $\mathbb{C}^{3} / \mathbb{Z}_{3}$ singularity studied in the previous section. Thus, a sensible resolution of $T^{6} / \mathbb{Z}_{3}$ can be constructed by cutting an open patch around each singularity and replacing it with the smooth space studied above.

To perform this procedure in detail one has to face the following complicating issues: first of all one has to worry whether the gluing process can be carried out properly. Constructing the blow-up of $T^{6} / \mathbb{Z}_{3}$ by naively joining 27 resolutions of $\mathbb{C}^{3} / \mathbb{Z}_{3}$ with finite volume seems to lead to a space that is not completely smooth. We ignore this complication by assuming that a more complicated smooth gluing procedure exists, and that for essentially topological questions (e.g. what models do exist and what are their spectra?) this procedure can be trusted. As we are not only gluing together the $\mathbb{C}^{3} / \mathbb{Z}_{3}$ 
blow-ups but also the bundles on them, we have to confirm that the resulting bundle on the resolution of $T^{6} / \mathbb{Z}_{3}$ actually exists. There are two different ways of analyzing this: we can check various consistency conditions ensuring the existence or, from the orbifold point of view, we have to show that $\mathrm{F}$ - and $\mathrm{D}$-flat directions are allowed by the (super)potential of the compact orbifold theory.

To systematically investigate these issues, we first show that resolutions of compact orbifold models without Wilson lines are possible. Next, we review properties of $\mathbb{Z}_{3}$ orbifold models with Wilson lines and their resolutions. We finish this section by two examples: the first example considers the blow-up of an orbifold with a single Wilson line, illustrating the gluing procedure of the gauge bundle. The second one examines an orbifold with two Wilson lines and defines an MSSM-like model. Therefore, it is phenomenologically interesting to see whether this model can exist in the blow-up.

\subsection{Resolution of the $T^{6} / \mathbb{Z}_{3}$ orbifolds without Wilson lines}

To obtain the smooth resolution of an orbifold without Wilson lines, the first possibility is to choose the same $\mathrm{U}(1)$ bundle embedding at each fixed point. In such a case, the local consistency conditions are enough to guarantee the existence of the bundle. Indeed, the only extra conditions on the bundle would come from the Bianchi identity integrated on the new compact 4-cycles, which are generated by the gluing and thus "inherited" from $T^{6}$. On the other hand, these new 4-cycles are obtained by combining the non-compact 4-cycles of the resolved $\mathbb{C}^{3} / \mathbb{Z}_{3}$ singularities. However, for this resolution (see [15, 18]), the local Bianchi identity on $\mathbb{C}^{3} / \mathbb{Z}_{3}$ implies the Bianchi identity on these non-compact 4cycles. Thus, the local consistency conditions ensure that the new consistency conditions, due to the gluing, are satisfied. Therefore, all local models can be naturally extended to global ones, with spectra given by 27 copies of the local spectra. On the orbifold, this resolution is characterized by requiring that identical twisted states at all fixed points acquire non-vanishing vevs of the same magnitude and identical orientation.

From the orbifold perspective, it requires a little more work to show that this blowup exists. D-flatness does not constitute a problem: the auxiliary field $D^{a}$ is simply the sum of the local fixed point contributions $D^{(f) a}$. Since at all fixed points identical twisted states, the blow-up modes, attain exactly the same vev, the individual D-terms $D^{(f) a}$ are all the same. For the compact models investigated here all D-terms vanish, except possibly the ones associated with the local anomalous U(1)'s, analogously to the noncompact models studied before. For the anomalous U(1)'s the same comment holds as for the non-compact situation, see subsection 2.7.

F-flatness of the compact blow-up does not automatically follow from $\mathrm{F}$-flatness of the local $\mathbb{C}^{3} / \mathbb{Z}_{3}$ blow-ups, because the superpotential of the compact orbifold is much richer than its non-compact counterpart. Of course, all local fixed point couplings that were allowed on $\mathbb{C}^{3} / \mathbb{Z}_{3}$ are still allowed. But since the $R$-symmetry group is reduced in the transition from the non-compact to the compact orbifold as $\mathrm{U}(3)_{R} \rightarrow \mathbb{Z}_{3}^{3}$, new local interactions at a single fixed point can appear. Moreover, there is the possibility of non-local interactions involving twisted states living at different fixed points. 
Most arguments in subsection 2.5 were based on the existence of certain gauge invariant operators and therefore do still apply in the compact case. For example, the blow-up $\mathrm{A} \rightarrow \mathrm{AI}$ exists because (18) yields vanishing $\mathrm{F}$-terms for all fields if only the singlet gets a vev. In the compact case we have to take non-local interactions into account,

$$
\mathcal{W} \sim \sum_{f, g, h} 27^{(f)} \times 27^{(g)} \times 27^{(h)} \sim \sum_{f, g, h} 16_{-1}^{(f)} \times 16_{-1}^{(g)} \times 10_{2}^{(h)}+10_{2}^{(f)} \times 10_{2}^{(g)} \times 1_{-4}^{(h)},
$$

where the sum over the different fixed points $f, g, h$ is restricted by the space group selection rule [16, 26]. Because at all fixed points only the singlets $\mathbf{1}_{-4}^{(f)}$ get vevs, all Fterms still vanish. Hence, we only have to worry about gauge invariant superpotential terms that do not have an analog on the non-compact orbifold.

The only case where new (and relevant) interactions arise on the compact orbifold, which did not exist in the non-compact version, is the standard embedding AS. Because of the reduction of the $R$-symmetry group to $\mathbb{Z}_{3}^{3}$ there is now a cubic gauge invariant term in the superpotential

$$
\mathcal{W} \sim \sum_{\alpha, f, g, h} \epsilon^{k l m} G_{k}^{(f) \alpha} G_{l}^{(g) \alpha} G_{m}^{(h) \alpha}+\ldots
$$

As argued in [16], this superpotential allows the same F-flat vev as in the non-compact case: $G=v \mathbf{1}$.

This analysis shows that a simultaneous blow-up of all 27 fixed points, where the same blow-up mode at each fixed point acquires the same non-vanishing vev, allows for D- and F-flatness. It is therefore possible - and straightforward - to construct consistent resolutions of compact orbifolds from the resolutions of the local ones, which were studied in the previous sections.

\subsection{Orbifolds with Wilson lines}

Even though the description of orbifold models with Wilson lines is well-known [35], we give here a detailed review to be able to emphasize similarities as well as differences compared to the description of blow-ups in the next subsection.

In compact orbifold models with multiple singularities, there can be different gauge embedding shifts $V_{\text {orb }}^{(f)}$ at each fixed point $f$. Each of these shifts satisfies the local version of the modular invariance requirement

$$
\left(V_{\text {orb }}^{(f)}\right)^{2}=0 \bmod 6 .
$$

This means that, in the case of $T^{6} / \mathbb{Z}_{3}$, the model is locally completely determined by the gauge groups and spectra listed in Table 3 .

The possibility of having different local gauge shifts can also be encoded in the language of discrete Wilson lines defined as $A_{\text {orb }}^{(f g)}=V_{\text {orb }}^{(g)}-V_{\text {orb }}^{(f)}$ among two fixed points $f$ and $g$. However, not all local shifts $V_{\text {orb }}^{(f)}$ are independent due to geometrical constraints. 
As is well-known [37], any local shift $V_{\text {orb }}^{(f)}$ can be represented as $V_{\text {orb }}^{(f)} \equiv V_{\text {orb }}+f_{i} A_{\text {orb }}^{(i)}$, where we define a global orbifold shift $V_{\text {orb }}=V_{\text {orb }}^{(0)}$ and the three discrete Wilson lines $A_{\text {orb }}^{(i)}=V_{\text {orb }}^{(i)}-V_{\text {orb }}^{(0)}$ of the $\mathbb{Z}_{3}$ orbifold in the three complex directions 5 The $\equiv$ symbol means that the two sides of the equation are equal up to $3 \Lambda$, where $\Lambda$ is a generic element of the root lattice of $\mathrm{E}_{8} \times \mathrm{E}_{8}^{\prime}$. These vectors satisfy $\mathbb{Z}_{3}$ periodicities

$$
3 V_{\text {orb }} \equiv 3 A_{\text {orb }}^{(i)} \equiv 0
$$

and the rewritten modular invariance conditions

$$
\left(V_{\text {orb }}\right)^{2}=0 \bmod 6, \quad\left(A_{\text {orb }}^{(i)}\right)^{2}=0 \bmod 6, \quad 2 V_{\text {orb }} A_{\text {orb }}^{(i)}=0 \bmod 6 .
$$

Sitting at a fixed point $\left(f_{1}, f_{2}\right)$ of the first two tori but freely moving in the third one these conditions imply

$$
V_{\text {orb }}^{\left(f_{1}, f_{2}, 0\right)}+V_{\text {orb }}^{\left(f_{1}, f_{2}, 1\right)}+V_{\text {orb }}^{\left(f_{1}, f_{2}, 2\right)} \equiv 0
$$

Similar conditions have to be imposed for the other choices of tori.

At each fixed point the local action $V_{\text {orb }}^{(f)}$ generates a (different) gauge symmetry breaking. The resulting 4D gauge group is the one surviving all local projections simultaneously. Using the splitting of the generators of $\mathrm{E}_{8} \times \mathrm{E}_{8}^{\prime}$ into Cartan elements $H^{I}$ and other elements $E^{p}$, where $p$ denotes the 16 -dimensional root vector of $E^{p}$, such that $\left[H^{I}, E_{p}\right]=p^{I} E_{p}$, the effective $4 \mathrm{D}$ gauge group is determined by $V_{\text {orb }}^{(f)} \cdot p=0 \bmod 3$ for each fixed point $f$. These conditions can be rewritten in terms of the gauge shift and Wilson lines as

$$
V_{\text {orb }} \cdot p=0 \bmod 3, \quad A^{(i)} \cdot p=0 \bmod 3, \text { for } i=1,2,3,
$$

and provide an efficient way of characterizing the effective 4D gauge group.

We have to distinguish between localized and delocalized matter when describing the spectrum on $T^{6} / \mathbb{Z}_{3}$. The twisted states localized in the fixed points are organized into representations of the larger gauge group at the respective fixed point, determined by $V_{\text {orb }}^{(f)}$ only. They are listed in Table 3 . Since $T^{6} / \mathbb{Z}_{3}$ has no fixed planes or lines, only untwisted matter is delocalized 6 It feels the action of all local projections.

\subsection{The resolution of $T^{6} / \mathbb{Z}_{3}$ models with Wilson lines}

In this section we describe how to construct smooth resolutions of compact orbifold models in the presence of discrete Wilson lines. After summarizing the basic matching principle, we study the consistency conditions that must be enforced due to the global

\footnotetext{
${ }^{5}$ With slight stretch of notation we use $i=\left(\delta_{1 i}, \delta_{2 i}, \delta_{3 i}\right)$ to indicate the fixed point which lies in the $i$ th complex $T^{2}$.

${ }^{6}$ This is not generically true; most orbifolds have sectors of delocalized twisted matter, e.g. the second twisted sectors in some $T^{6} / \mathbb{Z}_{2 n}$ orbifolds.
} 


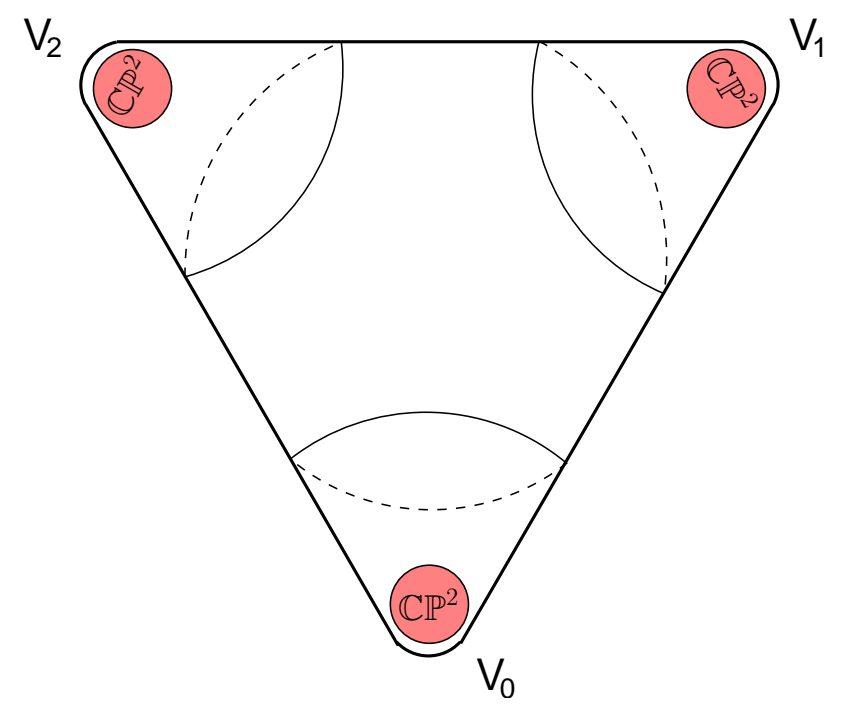

Figure 3: A schematic two dimensional cross section of the resolved $T^{6} / \mathbb{Z}_{3}$ orbifold is depicted. The fixed points are replaced by smooth surfaces that contain $\mathbb{C P}^{2}$ 's.

properties of the compact space. Finally, we explain how to compute the spectrum of the resolved models.

Having discrete Wilson lines on an orbifold essentially corresponds to wrapping different local fluxes on the $\mathbb{C P}^{2}$ 's inside the resolved space, i.e. choosing different embedding vectors $V^{(f)}$ at different resolved singularities. A schematic picture of the resolved situation is depicted in Fig. 3. Constraints on the possible fluxes come from the local Bianchi identities, related to the localized 4-cycles corresponding to the exceptional divisors: at each fixed point $f$ we have a condition 7

$$
\left(V^{(f)}\right)^{2}=12 .
$$

Moreover, new conditions are due to the fact that the gauge bundles are not localized, but rather extend over the whole space. Hence, the gluing of different patches requires the various gauge backgrounds on non-trivial overlaps to be related in a consistent way. Therefore, we consider open patches $U^{(f)}$ and $U^{(g)}$ around the resolutions of orbifold singularities labeled by $f$ and $g$ with gauge configurations $\mathfrak{U}_{1}^{(f)}$ and $\mathfrak{U}_{1}^{(g)}$, respectively. The transition function $g^{(f g)}=\left(g^{(g f}\right)^{-1}$ describes the relation between the two gauge one-form potentials on the intersection of the two patches:

$$
\mathfrak{U}_{1}^{(g)}=g^{(g f)}\left(\mathfrak{U}_{1}^{(f)}+\mathrm{d}\right) g^{(f g)} .
$$

Given a point where (any) three patches $f, g$ and $h$ overlap, we need $g^{(f g)} g^{(g h)} g^{(h f)}=1$. Moreover, we can identify the transition function $g^{(f g)}$ in the case of a U(1) gauge bundle with a function $A^{(f g)}$ between the two fixed points $f$ and $g$ as

$$
g^{(f g)}=e^{2 \pi i A^{(f g) I} H_{I} / 3} .
$$

\footnotetext{
${ }^{7}$ As explained in the previous section, the new conditions due to the presence of new compact 4-cycles are automatically satisfied once the local conditions are.
} 
The function $A^{(f g)}$ is generically not constant. However, in the blow-down limit it becomes constant and can be identified with a discrete Wilson line $A_{\text {orb }}^{(f g)}$ on the orbifold between the fixed points $f$ and $g$. In this limit, we have $A^{(f g)} \equiv A_{\text {orb }}^{(f g)}$ and for that reason we may refer to the function $A^{(f g)}$ as a Wilson line on the resolved space.

The co-cycle condition $g^{(f g)} g^{(g h)} g^{(h f)}=1$ can be expressed in terms of the Wilson lines as

$$
A^{(f g)}+A^{(g h)}+A^{(h f)} \equiv 0 .
$$

This condition applies to any manifold. It states conditions for the existence of a flux in the case a space cannot be covered with a single open patch.

The construction of such Abelian gauge bundles on the resolution obtained from gluing the local patches, as discussed above, leads to a more general class of models than those that are obtained as blow-ups of global orbifold models: the gauge bundle on the resolution of $T^{6} / \mathbb{Z}_{3}$ descents down to the orbifold gauge bundle only if an identification

$$
V^{f} \equiv V_{o r b}^{f}
$$

can be made at each of the resolved fixed points of $T^{6} / \mathbb{Z}_{3}$, as explained in (15). Thus, the geometric condition (42) has to be imposed on the resolution shifts, too, and we have

$$
V^{\left(f_{1}, f_{2}, 0\right)}+V^{\left(f_{1}, f_{2}, 1\right)}+V^{\left(f_{1}, f_{2}, 2\right)} \equiv 0
$$

and corresponding expressions after permutations of the tori. Resolution models, that do not satisfy this condition, nevertheless define valid supergravity compactifications, even though they can not be associated with a global orbifold model. This shows that such global orbifold constructions may lead only to a restrictive class of models. Since the motivation of this paper is to study the blow-up of such global orbifolds, we enforce the conditions (49).

Given the consistency conditions on the bundles, we can study how to compute the spectra in the 4D models. Gauge bosons of the global unbroken 4D gauge group are distributed over the whole resolution manifold. Let us consider the blow-up of two singularities $f$ and $g$, each surrounded by an open patch. Assume that the patches have a non-vanishing overlap. Again, we consider gauge configurations $\mathfrak{U}_{1}^{(f)}$ and $\mathfrak{U}_{1}^{(g)}$ on the patches $U^{(f)}$ and $U^{(g)}$, respectively. Since we are interested in the resulting zero modes, we can assume that the non-trivial topology is encoded in the background gauge configurations $\mathcal{A}_{1}^{(f)}$ and $\mathcal{A}_{1}^{(g)}$ only, i.e. they satisfy the same relation as above

$$
\mathcal{A}_{1}^{(g)}=g^{(g f)}\left(\mathcal{A}_{1}^{(f)}+\mathrm{d}\right) g^{(f g)} .
$$

The full gauge configurations $\mathfrak{U}_{1}^{(f)}$ is written as a sum of the background $\mathcal{A}_{1}^{(f)}$ plus perturbations $A_{1}^{(f)}$ :

$$
\mathfrak{U}_{1}^{(f)}=\mathcal{A}_{1}^{(f)}+A_{1}^{(f)} .
$$

In this expansion we only take the untwisted modes into account. This means that we find

$$
A_{1}^{(g)}=g^{(g f)} A_{1}^{(f)} g^{(f g)} \Rightarrow\left\{\begin{array}{l}
A_{1}^{(g) I}=A_{1}^{(f) I} \\
A_{1}^{(g) p}=e^{2 \pi i A_{f g}^{I} p_{I}} A_{1}^{(f) p}
\end{array}\right.
$$


after expanding the perturbations as $A_{1}^{(f)}=A_{1}^{(f) I} H_{I}+A_{1}^{(f) p} E_{p}$, with the notation of the $H_{I}$ and $E_{p}$ explained above (43). If we assume the overlap region of the two patches $U^{(f)}$ and $U^{(g)}$ to be far away from the blown up singularities, the zero modes of the perturbations are essentially constant modes. Because the constant zero modes on both sides of the gluing region $U^{(f)} \cap U^{(g)}$ can be connected and stay a zero mode, they simply have to be equal. This means that the phase must be trivial: $A^{(f g)} \cdot p=0$. Hence, in terms of bundle shift $V$ and Wilson lines $A^{(f)}=A^{(f 0)}$ we find the projection conditions

$$
V \cdot p=0, \quad A^{(f)} \cdot p=0, \text { for } f \neq 0 .
$$

As compared to the maximally four projection conditions for the effective 4D gauge group on the orbifold, we see that there are generically more and stronger conditions on the surviving $4 \mathrm{D}$ gauge group on the resolution.

The main reason for the additional gauge symmetry breaking on the resolution is that the conditions (53) are not "mod 3", as they were in the orbifold case. This means that we cannot neglect (triple multiples of) $\mathrm{E}_{8} \times \mathrm{E}_{8}$ lattice vectors and reduce to four projections at most. In particular, this implies that an orbifold irrelevant Wilson line, i.e. just being three times an $\mathrm{E}_{8} \times \mathrm{E}_{8}$ lattice vector, can have a non-trivial effect on the resolution gauge group. In this case the same $\mathrm{U}(1)$ bundle is chosen at each fixed point, but they are differently aligned in the $\mathrm{E}_{8} \times \mathrm{E}_{8}^{\prime}$. From the orbifold point of view this choice corresponds to identical twisted states at all fixed points acquiring non-vanishing vevs of the same magnitude, but different orientation. It will also be shown later that this can help to ensure D-flatness for all U(1)'s.

As an example of this situation we can consider the gauge embeddings $V=$ $\left(-2^{3}, 0^{5}\right)\left(0^{8}\right)$ and $V^{\prime}=\left(1^{3}, 3,0^{4}\right)\left(0^{8}\right)$. They are both consistent and give rise to the same resolution model labeled as $\mathrm{AI}$ with gauge group $\mathrm{SO}(10) \times \mathrm{U}(3) \times \mathrm{E}_{8}$. It is the resolution of the orbifold model A with gauge group $\mathrm{E}_{6} \times \mathrm{SU}(3) \times \mathrm{E}_{8}$. Nevertheless, if we consider a compact model with one resolved singularity equipped with a $V$ - and another one with a $V^{\prime}$-embedding, such that the trivial Wilson line $A=\left(3^{4}, 0^{4}\right)\left(0^{8}\right)$ relates them, then the resulting gauge group is not $\mathrm{SO}(10) \times \mathrm{U}(3) \times \mathrm{E}_{8}$, but rather $\mathrm{SO}(8) \times \mathrm{U}(1) \times \mathrm{U}(3) \times \mathrm{E}_{8}$. This can also be confirmed from the orbifold perspective, when the 27 -plets of these two fixed points develop vevs for different components.

Finally, we describe the consequence of this for the matter on the blow-up of $T^{6} / \mathbb{Z}_{3}$ with Wilson lines. Locally, the delocalized matter was identified by the fact that it has a fractional multiplicity factor, $\frac{1}{9}$ (or multiples), see section 2.4. Because it is distributed over all patches, it feels projection conditions due to the transition functions between the patches. Thus, given a resolved singularity, say 0 , we have to impose

$$
A^{(f)} \cdot p=0 \bmod 3, \text { for } f \neq 0
$$

on its delocalized matter. The localized matter, with integral multiplicity, does not reach the overlap regions with the other patches and therefore feels no further projection conditions. Hence, the matter representations of the localized matter just branch with respect to the global unbroken $4 \mathrm{D}$ gauge group. 


\begin{tabular}{|c|c|c|c|c|}
\hline \multirow{2}{*}{$\begin{array}{c}\text { fixed point } \\
\text { (loc.) gauge group }\end{array}$} & \multicolumn{3}{|c|}{ matter } & \multirow{2}{*}{$\begin{array}{ll}\text { decomposition } & \text { field } \\
\text { to blow-up group } & \text { redefinition }\end{array}$} \\
\hline & mult. & local matt. & 4D matt. & \\
\hline $\begin{array}{c}\text { U Sector } \\
\mathrm{E}_{6} \times \mathrm{SO}(14) \times \mathrm{U}(1)^{3}\end{array}$ & 3 & & $(\mathbf{2 7}, \mathbf{1})_{(2,2,0)}$ & $\begin{array}{l}(\mathbf{1 6}, \mathbf{1})_{(2,2,0,-1)} \\
(\mathbf{1 0}, \mathbf{1})_{(2,2,0,2)} \\
(\mathbf{1}, \mathbf{1})_{(2,2,0,-4)}\end{array}$ \\
\hline \multirow{3}{*}{$\begin{array}{c}g_{1}=(\theta, 0) \\
\mathrm{E}_{7} \times \mathrm{SO}(14) \times \mathrm{U}(1)^{2}\end{array}$} & 1 & $(\mathbf{1}, \mathbf{1 4})_{(2,0)}$ & $(\mathbf{1}, \mathbf{1 4})_{(2,0,0)}$ & $(\mathbf{1}, \mathbf{1 4})_{(2,0,0,0)}=e^{-\frac{1}{2} T_{1}}(\mathbf{1}, \mathbf{1 4})_{(0,0,0,0)}^{m}$ \\
\hline & 1 & $(\mathbf{1}, \mathbf{1})_{(-4,0)}$ & $(\mathbf{1}, \mathbf{1})_{(-4,0,0)}$ & $(\mathbf{1}, \mathbf{1})_{(-4,0,0,0)}=v_{1} e^{T_{1}}$ \\
\hline & 3 & $(\mathbf{1}, \mathbf{1})_{(0,4)}$ & $(\mathbf{1}, \mathbf{1})_{(0,2,-2)}$ & $(\mathbf{1}, \mathbf{1})_{(0,2,-2,0)}=e^{T_{1}}(\mathbf{1}, \mathbf{1})_{(4,2,-2,0)}$ \\
\hline local blow-up at $g_{1}$ & \multicolumn{4}{|l|}{$\mathrm{CI}$} \\
\hline \multirow[t]{2}{*}{$\begin{array}{c}g_{2}=\left(\theta, e_{1}\right) \\
\mathrm{E}_{6} \times \mathrm{SU}(3) \times \mathrm{E}_{8}\end{array}$} & 1 & $(27,1,1)$ & $(\mathbf{2 7}, \mathbf{1})_{(0,0,0)}$ & $\begin{array}{l}(\mathbf{1 6}, \mathbf{1})_{(0,0,0,-1)}=e^{T_{2}}(\mathbf{1 6}, \mathbf{1})_{(0,0,0,3)} \\
(\mathbf{1 0}, \mathbf{1})_{(0,0,0,2)}=e^{-\frac{1}{2} T_{2}}(\mathbf{1 0}, \mathbf{1})_{(0,0,0,0)}^{m} \\
(\mathbf{1}, \mathbf{1})_{(0,0,0,-4)}=v_{2} e^{T_{2}}\end{array}$ \\
\hline & 3 & $(\mathbf{1}, \mathbf{3}, \mathbf{1})$ & $\begin{array}{l}(\mathbf{1}, \mathbf{1})_{(-2,-2,0)} \\
(\mathbf{1}, \mathbf{1})_{(0,2,2)} \\
(\mathbf{1}, \mathbf{1})_{(2,0,-2)}\end{array}$ & $\begin{array}{l}(\mathbf{1}, \mathbf{1})_{(-2,-2,0,0)}=e^{T_{2}}(\mathbf{1}, \mathbf{1})_{(-2,-2,0,4)} \\
(\mathbf{1}, \mathbf{1})_{(0,2,2,0)}=e^{T_{2}}(\mathbf{1}, \mathbf{1})_{(0,2,2,4)} \\
(\mathbf{1}, \mathbf{1})_{(2,0,-2,0)}=e^{T_{2}}(\mathbf{1}, \mathbf{1})_{(2,0,-2,4)}\end{array}$ \\
\hline local blow-up at $g_{2}$ & \multicolumn{4}{|l|}{$\mathrm{AI}$} \\
\hline \multirow{3}{*}{$\begin{array}{c}g_{3}=\left(\theta, e_{1}+e_{2}\right) \\
\mathrm{E}_{7} \times \mathrm{SO}(14) \times \mathrm{U}(1)^{2}\end{array}$} & 1 & $(\mathbf{1}, \mathbf{1 4})_{(0,2)}$ & $(\mathbf{1}, \mathbf{1 4})_{(0,2,0)}$ & $(\mathbf{1}, \mathbf{1 4})_{(0,2,0,0)}=e^{-\frac{1}{2} T_{3}}(\mathbf{1}, \mathbf{1 4})_{(0,0,0,0)}^{m}$ \\
\hline & 1 & $(\mathbf{1}, \mathbf{1})_{(0,-4)}$ & $(\mathbf{1}, \mathbf{1})_{(0,-4,0)}$ & $(\mathbf{1}, \mathbf{1})_{(0,-4,0,0)}=v_{3} e^{T_{3}}$ \\
\hline & 3 & $(\mathbf{1}, \mathbf{1})_{(4,0)}$ & $(\mathbf{1}, \mathbf{1})_{(2,0,2)}$ & $(\mathbf{1}, \mathbf{1})_{(2,0,2,0)}=e^{T_{3}}(\mathbf{1}, \mathbf{1})_{(2,4,2,0)}$ \\
\hline local blow-up at $g_{3}$ & \multicolumn{4}{|l|}{ CI } \\
\hline
\end{tabular}

Table 5: This table gives an overview of the complete global 4D spectrum of the blown up orbifold theory. The field redefinitions necessary to have precisely local matching between the orbifold blow-up theory and the resolution model are indicated. The $\mathrm{U}(1)^{4}$-generators of the $4 \mathrm{D}$ gauge group in blow-up are $Q_{1}=\left(2,2,0^{6}\right)\left(2,0^{7}\right), Q_{2}=\left(2,0,-2,0^{5}\right)\left(-2,0^{7}\right)$, $Q_{3}=\left(0,-2,-2,0^{5}\right)\left(2,0^{7}\right)$ and $Q_{4}=\left(2,-2,2,0^{5}\right)\left(0^{8}\right)$. There are two anomalous combinations: $Q_{1}^{a n}=Q_{1}+Q_{2}$ and $Q_{2}^{a n}=Q_{4}$.

\subsection{One Wilson line model with three anomalous U(1)'s}

In the following we give a specific example of an orbifold model in the presence of a discrete Wilson line, and study one of its blown up versions. On the resolution the model has three anomalous U(1)'s. The bulk universal and the local model-dependent axions are all involved in the anomaly cancellation.

To make the general discussion more explicit, we consider the model obtained from the $T^{6} / \mathbb{Z}_{3}$ orbifold with gauge shift $V_{\text {orb }}=\left(2,2,0^{6}\right)\left(2,0^{7}\right)$ and one Wilson line $A_{\text {orb }}=$ $\left(0,-4,2,0^{5}\right)\left(-2,0^{7}\right)$ in the first complex torus. 8 First we look at the orbifold and then investigate its resolution. Due to the Wilson line on the orbifold, the 27 fixed points are grouped together in three sets of nine fixed points each. The three sets are characterized

\footnotetext{
${ }^{8}$ For recent work about the computation of orbifold spectra with Wilson lines see for example [5, 38.
} 
by the local shift vectors $V_{\text {orb }}, V_{\text {orb }}+A_{\text {orb }}$ and $V_{\text {orb }}+2 A_{\text {orb }}$ respectively. The same local gauge group and charged matter is present at all nine fixed points of each set. Details are given in Table 5, where representatives of the three sets of fixed points are identified by their space group representatives $g_{1}, g_{2}$ and $g_{3}$, respectively.

The next task is to find a resolution model that, in the blow down limit, reduces to this orbifold model. We find that at the $g_{1}$ singularities, we have to choose the CI resolution, with gauge bundle defined by the blow-up shift $V_{1}=V_{\text {orb }}$; at the $g_{2}$ singularities the AI resolution, with $V_{2}=V_{\text {orb }}+A_{\text {orb }}$. Finally, at the $g_{3}$ singularities we have to choose again resolution CI, but with a different shift $V_{3}=V_{\text {orb }}+2 A_{\text {orb }}+3 \Lambda$, where $3 \Lambda=\left(0,6,-6,0^{5}\right)\left(0^{8}\right)$ represents, from the orbifold perspective, an irrelevant Wilson line, which is nevertheless crucial to ensure that $V_{3}$ satisfies the local Bianchi identity. This "irrelevant" Wilson line leads to additional gauge symmetry breaking on the resolution. The local gauge group and the chiral matter on each of the three sets of nine patches can be found in table 3 . The different bundle vectors $V_{1}, V_{2}$ and $V_{3}$ combined lead to further symmetry breaking of the local gauge groups at the 27 resolved fixed points to the global $4 \mathrm{D}$ gauge group:

$$
\mathrm{SO}(10) \times \mathrm{SO}(14) \times \mathrm{U}(1)^{4}
$$

Consequently, the representations of the local spectrum on each of the different fixed point resolutions becomes

$$
\begin{array}{lll}
g_{1}: C I: & \frac{1}{9}\left[(\mathbf{1 6} ; \mathbf{1})_{(2,2,0,-1)}+(\mathbf{1 0} ; \mathbf{1})_{(2,2,0,2)}+(\mathbf{1} ; \mathbf{1})_{(2,2,0,-4)}\right]+3(\mathbf{1} ; \mathbf{1})_{(4,2,-2,0)}, \\
g_{2}: A I: \quad & \frac{1}{9}\left[(\mathbf{1 6} ; \mathbf{1})_{(2,2,0,-1)}+(\mathbf{1 0} ; \mathbf{1})_{(2,2,0,2)}+(\mathbf{1} ; \mathbf{1})_{(2,2,0,-4)}\right]+(\mathbf{1 6} ; \mathbf{1})_{(0,0,0,3)} \\
& +3\left[(\mathbf{1} ; \mathbf{1})_{(-2,-2,0,4)}+(\mathbf{1} ; \mathbf{1})_{(0,2,2,4)}+(\mathbf{1} ; \mathbf{1})_{(2,0,-2,4)}\right] \\
& \quad \frac{1}{9}\left[(\mathbf{1 6} ; \mathbf{1})_{(2,2,0,-1)}+(\mathbf{1 0} ; \mathbf{1})_{(2,2,0,2)}+(\mathbf{1} ; \mathbf{1})_{(2,2,0,-4)}\right]+3(\mathbf{1} ; \mathbf{1})_{(2,4,2,0)} .
\end{array}
$$

Comparing this with Table 3 , the localized states (with integral multiplicities) are simply branched to representations of the unbroken 4D gauge group, while some delocalized states (with multiplicity 1/9) are projected out. Because these delocalized states live everywhere on the compact resolution, their spectra at the three types of patches are all the same. The complete resolution spectrum is obtained by multiplying each line of (56) by nine.

We can also study this resolved model from the orbifold blow-up perspective: we select a single twisted field per fixed point that attains a vev chosen along a F-flat direction, but some D-terms are induced in order to match the FI-terms of the resolution 9 We determine the gauge symmetry breaking induced by this. Each set of singularities $g_{i}$

\footnotetext{
${ }^{9}$ For complete $\mathrm{F}-$ and $\mathrm{D}$-flatness, we can choose another vacuum configuration, defined by the monomial $(\mathbf{2 7}, \mathbf{1})_{(2,2,0)}^{2}(\mathbf{1}, \mathbf{1})_{(-4,0,0)}(\mathbf{2 7}, \mathbf{1})_{(0,0,0)}(\mathbf{1}, \mathbf{1})_{(0,-4,0)}$. This means that the additional untwisted field $(\mathbf{2 7}, \mathbf{1})_{(2,2,0)}$ gets a vev leading to a further gauge symmetry break down.
} 
has a different blow-up mode and gauge symmetry breaking:

$$
\begin{aligned}
& g_{1}:\left\langle(\mathbf{1} ; \mathbf{1})_{(-4,0,0)}\right\rangle \neq 0: \quad \mathrm{E}_{7} \times \mathrm{SO}(14) \times \mathrm{U}(1)^{2} \rightarrow \mathrm{E}_{7} \times \mathrm{SO}(14) \times \mathrm{U}(1), \\
& g_{2}:\left\langle(\mathbf{2 7} ; \mathbf{1})_{(0,0,0)}\right\rangle \neq 0: \quad \mathrm{E}_{6} \times \mathrm{SU}(3) \times \mathrm{E}_{8} \rightarrow \mathrm{SO}(10) \times \mathrm{U}(3) \times \mathrm{E}_{8}, \\
& g_{3}:\left\langle(\mathbf{1} ; \mathbf{1})_{(0,-4,0)}\right\rangle \neq 0: \quad \mathrm{E}_{7} \times \mathrm{SO}(14) \times \mathrm{U}(1)^{2} \rightarrow \mathrm{E}_{7} \times \mathrm{SO}(14) \times \mathrm{U}(1) .
\end{aligned}
$$

The global 4D gauge group can be obtained as the intersection of the three local ones, and coincides with the one given in (55). By performing the appropriate field redefinitions on the orbifold, given in Table 5, the blown-up orbifold and the smooth resolution model match perfectly.

Let us finally comment on the issue of anomalous U(1)'s of this orbifold model in blow-up. As one can see from Table 5, at each fixed point the blow-up mode induces a localized axion. We refer to these axion superfields as $T_{1}, T_{2}$ and $T_{3}$, depending on which set of nine fixed points they belong to. Together with $b_{2}$, there can in principle be four independent types of axions in the resolution model; this theory could maximally accommodate four anomalous U(1)'s. Because the anomaly polynomial

$$
\begin{gathered}
\hat{I}_{6}^{r e s}=216 F_{1}^{a n}\left(\frac{3}{2}\left(F_{1}^{a n}\right)^{2}+\left(F_{2}^{a n}\right)^{2}+\frac{1}{2}\left(F^{n o}\right)^{2}+\frac{1}{24} F_{10}^{2}-\frac{1}{32} \operatorname{tr} R^{2}\right) \\
+216 F_{2}^{a n}\left(\frac{3}{2}\left(F_{1}^{a n}\right)^{2}+7\left(F_{2}^{a n}\right)^{2}+\frac{1}{2}\left(F^{n o}\right)^{2}+\frac{1}{8} F_{10}^{2}-\frac{7}{96} \operatorname{tr} R^{2}\right)
\end{gathered}
$$

with $F^{n o}=F_{1}-F_{2}-2 F_{3}$, is a sum of two factorized pieces, we could infer that there are only two anomalous U(1)'s, $F_{1}^{a n}=F_{1}+F_{2}$ and $F_{2}^{a n}=F_{4}$. (The corresponding charges are defined in the caption of Table 5.) However, if we more physically define the number of anomalous U(1)'s as the number of independent massive U(1) gauge fields, the number is three: three different vevs $v_{1}, v_{2}$ and $v_{3}$ break the $\mathrm{U}(1)$ symmetries $Q_{1}, Q_{4}$ and $Q_{2}$, respectively. The three axions $T_{1}, T_{2}$ and $T_{3}$ that do transform under three different combinations of the U(1)'s couple to the corresponding gauge field strengths, leading to three massive gauge fields. We can confirm this statement directly on the resolution by considering the gauge transformations

$$
\delta_{\Lambda} b_{0}^{g_{1}}=-2 \operatorname{tr}\left[Q_{1} \Lambda\right], \quad \delta_{\Lambda} b_{0}^{g_{2}}=-2 \operatorname{tr}\left[Q_{4} \Lambda\right], \quad \delta_{\Lambda} b_{0}^{g_{3}}=-2 \operatorname{tr}\left[Q_{2} \Lambda\right],
$$

obtained from (32) for the local expansions of $B_{2}$ at the resolutions of the different fixed points. Hence, these states can be identified as

$$
b_{0}^{g_{i}}=2 T_{i}
$$

with the local axions $T_{i}$ from the orbifold blow-up.

\subsection{Can we blow-up a $\mathbb{Z}_{3}$ MSSM model?}

We consider the $\mathbb{Z}_{3}$ orbifold model with two Wilson lines initially introduced in [2]. This model is interesting because it was one of the first string models with Standard Model 
gauge group and three generations of quarks and leptons. A potential problem of this model is the set of vector-like exotics in the spectrum. Only if these exotic states can all be made heavy, the effective low energy spectrum will be identical to that of the MSSM. The way this may happen is by turning on appropriate vevs. As vevs of twisted states lead to blow-ups of the singularities on which they are localized, it is interesting to investigate blow-up versions of this model. Therefore, we assume that the blow-up of this model is generated by single vevs of twisted states at each of the 27 fixed points. This assumption guarantees that we can rely on the Abelian bundles, constructed in section 2.3 , only. We focus on the question whether crucial properties of the MSSM are maintained in blow-up.

The work of [2, 36] revealed the presence of two hypercharge candidates amongst the eight $\mathrm{U}(1)$ factors of the model and an resulting ambiguitiy of identifying the MSSM particle spectrum. However, for either choice the orbifold theory cannot be completely blown up without breaking hypercharge. To resolve all singularities simultaneously, one blow-up mode has to be chosen per fixed point. Table 1 of [36] implies that all the states at the fixed point $\left(n_{1}, n_{3}\right)=(-1,-1)$ carry the same charge under both hypercharge candidates. Hence, by blowing up this singularity, we inevitably break hypercharge. There is only one way to avoid the end of any phenomenology in this orbifold model in full blow-up: the Higgs doublet $H_{1}$ of the MSSM at $(-1,-1)$ has to obtain a vev. Hence, the blow-up procedure has the interpretation of electroweak symmetry breaking. As far as we have been able to confirm, such a scenario still does not lead to a phenomenologically acceptable situation, because the vanishing of all the D-terms requires the vev of $H_{1}$ to be of the order of the compactification scale, i.e. far too large.

For this reason we explore a second possibility and resolve all singularities except the one at $\left(n_{1}, n_{3}\right)=(-1,-1)$. This partial resolution can be performed in an entirely $\mathrm{F}$ - and D-flat way, in all $\mathrm{U}(1)$ directions including the anomalous one and without breaking the hypercharge. For F-flatness, we need higher orders in the superpotential to guarantee that the derivative of the superpotential has a zero. For concreteness, consider the situation in which the fields listed in Table 6] all have non-vanishing vevs. Their gauge invariant monomial

$$
h_{2}\left(h_{10}\right)^{2}\left(h_{14}\right)^{2} h_{15}\left(h_{17}\right)^{3} h_{21}\left(h_{23}\right)^{3}\left(h_{24}\right)^{2}
$$

corresponds to the following relation between the vevs [29]

$$
\sqrt{6} h_{2}=\sqrt{3} h_{10}=\sqrt{3} h_{14}=\sqrt{6} h_{15}=\sqrt{2} h_{17}=\sqrt{6} h_{21}=\sqrt{2} h_{23}=\sqrt{3} h_{24},
$$

which ensures D-flatness. In this configuration, the hypercharge is identified to be $Y=$ $\frac{1}{6}\left(\frac{1}{3} Q_{1}-\frac{1}{2} Q_{2}-Q_{3}+Q_{4}\right)$, so that none of the blow-up modes is charged under it. Since $H_{1}$ is massless but does not constitute a flat direction of the effective scalar potential away from this point (i.e. at least as long as supersymmetry is not broken), the Higgs cannot acquire a vev. Consequently, electroweak symmetry breaking can only occur at low energies. Furthermore, in this vev configuration all extra U(1)'s are broken and all extra colour triplets acquire high masses from trilinear couplings. However, some of the other vector-like exotics stay massless at this order in the superpotential. Thus finally, neither the singular orbifold nor the everywhere smooth resolution of all the fixed points, but the partial blow-up to this hybrid model can potentially save phenomenology. 


\begin{tabular}{|c|rr|rrrrrrrr|c|c|}
\hline state & \multicolumn{1}{|c|}{ fixed point } & \multicolumn{8}{|c|}{ U(1) charges } & hyper & local \\
label & $n_{1}$ & $n_{3}$ & $Q_{1}$ & $Q_{2}$ & $Q_{3}$ & $Q_{4}$ & $Q_{5}$ & $Q_{6}$ & $Q_{7}$ & $Q_{8}$ & charge $Y$ & blow-up \\
\hline \hline$h_{2}$ & 0 & 0 & -3 & -2 & 3 & 3 & -3 & 4 & 0 & 0 & 0 & DI \\
$h_{10}$ & 1 & 0 & -3 & -2 & 3 & 3 & 1 & -2 & 2 & -4 & 0 & BI \\
$h_{14}$ & -1 & 0 & 6 & 4 & 0 & 0 & 2 & 4 & -2 & -2 & 0 & BI \\
$h_{15}$ & 0 & 1 & -6 & 0 & 0 & 2 & -4 & 0 & -4 & 0 & 0 & DI \\
$h_{17}$ & 0 & -1 & 0 & -4 & 0 & -2 & -2 & -4 & 4 & 0 & 0 & CI \\
$h_{21}$ & 1 & 1 & -6 & 0 & 0 & 2 & 0 & 0 & 4 & -4 & 0 & CI \\
$h_{23}$ & -1 & 1 & 3 & 6 & -3 & -1 & 1 & 0 & 0 & 4 & 0 & DI \\
$h_{24}$ & 1 & -1 & 0 & -4 & 0 & -2 & 2 & -4 & 0 & -4 & 0 & CI \\
\hline
\end{tabular}

Table 6: The eight blow-up modes -one per resolved fixed point- are chosen to be singlets with respect to $\mathrm{SU}(3) \times \mathrm{SU}(2)_{L} \times \mathrm{U}(1)_{Y}$. The notation used here follows [36].

\subsection{F- and D-terms for compact blow-ups}

We have mainly focused on compact resolutions with multiple anomalous U(1)'s and corresponding FI-terms. From the orbifold perspective, we have seen that these terms can be interpreted as non-vanishing D-terms induced by vevs of the blow-up modes. This situation is exactly the same as explained in section 2.7. In the following, we will discuss various possibilities to obtain stable resolutions by finding orbifold blow-ups corresponding to vacua with $F=D=0$.

The first method was discussed in the previous section, where it was necessary to blow-up the orbifold only partially in order to obtain $F=D=0$. This may seem a rather easy way out. A more interesting possibility is that some additional matter fields, either twisted or untwisted, take non-vanishing vevs. When more than one twisted state develops vevs at a single fixed point, we expect a non-Abelian gauge background to be generated on the resolution, as discussed at the end of section 2.5. A vev for an untwisted state leads to a continuous Wilson line. An example of the latter case was presented in Section 3.4, where the vev of the untwisted state $(\mathbf{2 7}, \mathbf{1})_{(2,2,0)}$ yielded a stable vacuum.

The general idea of a third method is to perform different blow-ups of degenerate fixed-points, i.e. of fixed points not distinguished by Wilson lines from the orbifold perspective. This can be achieved by choosing different blow-up modes at the various fixed-points. They may be either contained in different types of non-Abelian representations or in the same ones, but in different components. This allows for choosing the vevs at the different fixed points such that all D-terms vanish globally.

We can exemplify the latter possibility by considering the blow-up of the compact orbifold B without Wilson lines, see Section 3.1. Here, the blow-up mode is contained in the representation $(\mathbf{1}, \mathbf{3} ; \mathbf{1}, \mathbf{3})$, denoted by the matrix $C$. D-flatness can be guaranteed by assigning a vev of the same magnitude, but different orientation to each of the fields $C_{i}$, localized at one of the 27 fixed points $i=1, \ldots, 27$. This corresponds to a gauge 
invariant monomial of the form

$$
\prod_{i=1}^{27} C_{i}
$$

breaking the $\mathrm{SU}(3)^{2}$ factors of the $4 \mathrm{D}$ orbifold gauge group to $\mathrm{U}(1)^{4}$. Furthermore, Fflatness $F=0$ can be achieved at isolated points using higher order couplings in the superpotential yielding stable SUSY preserving vacua.

\section{The resolution of the $\mathbb{C}^{2} / \mathbb{Z}_{2}$ orbifold}

Orbifold singularities of the form $\mathbb{C}^{n} / \mathbb{Z}_{n}$ are resolved by a generalization of the procedure given in the previous section. In this way, it is possible to approach the resolution of the $\mathbb{C}^{2} / \mathbb{Z}_{2}$ singularity that is phenomenologically relevant given that many appealing $4 \mathrm{D}$ orbifold models are based on compactifications on orbifolds having $\mathbb{C}^{2} / \mathbb{Z}_{2}$ subsectors.

In [15] the explicit form of the resolution curvature and bundles were given for the $\mathbb{C}^{2} / \mathbb{Z}_{2}$ singularity, as well as a study of the matching of $4 \mathrm{D}$ models arising from the $\mathrm{SO}(32)$ heterotic string quantized on the orbifold and on the resolution. We do not give the details of that derivation, rather, we summarize the relevant results in Table 7 . In the table we give the gauge group and spectra of the three orbifold models $2 \mathrm{~A}, 2 \mathrm{~B}$ and $2 \mathrm{C}$ in the first column. In the second column we list those of the three models 2AI, 2BI and 2CI obtained by compactifying 10D SO(32) supergravity on the resolution. Again, the multiplicities are fractional and multiples of $1 / 16$ for untwisted (non-localized) states, and are integer or half integer for twisted (localized) states. As in the $\mathbb{C}^{3} / \mathbb{Z}_{3}$ case, there is no direct matching of the spectra. They should be compared only after the blow-up mode has developed a vev. This vev induces a Higgs mechanism on the orbifold side of the matching partially breaking the gauge symmetry. In the mechanism, parts of the Higgs field are "eaten" by the gauge bosons becoming massive. From the resolution perspective, this can be seen in the multiplicities $7 / 8$ for the states corresponding to the Higgs fields. Indeed, such a multiplicity should be understood as an integer number (the old twisted field multiplicity) reduced by $1 / 8$, since $1 / 8$ of the twisted field is incorporated in the massive gauge fields 10 . All the other states match after the field redefinition given in the third column of Table 7 . No extra state becomes massive due to the fixed chirality of the hyper multiplets in $6 \mathrm{D}$.

The analysis of the matching at the pure spectrum level is thus not different from the $\mathbb{C}^{3} / \mathbb{Z}_{3}$ case. On the other hand, the study of the flatness of the blow-up mode, as well as that of anomaly cancellation, is technically very different. The first issue is due to the structural difference between $4 \mathrm{D}$ and $6 \mathrm{D}$ SUSY, the second one due to the fact that anomaly cancellation in 6D may proceed via two different diagrams, giving rise to different mechanisms: one is mediated by scalars (or four-forms in a dual picture), the other by two-forms. In the first situation the anomalous gauge boson gets a mass, in the second case it does not. We approach these aspects in the forthcoming sections.

\footnotetext{
${ }^{10}$ Note that the gauge bosons are delocalized, thus their multiplicity is $1 / 16$ times a factor of 2 , since they from doublets of the internal SU(2) holonomy.
} 


\begin{tabular}{|c|c|c|c|}
\hline matching & orbifold model & resolution model & field redefinitions \\
\hline \multirow{2}{*}{$\begin{array}{l}2 \mathrm{~A} \rightarrow 2 \mathrm{AI} \\
\begin{array}{l}\langle(\mathbf{2 8}, \mathbf{1}, \mathbf{2})\rangle \\
\quad \neq 0\end{array}\end{array}$} & $\mathrm{SO}(28) \times \mathrm{SU}(2)^{2}$ & $\mathrm{SO}(26) \times \mathrm{SU}(2) \times \mathrm{U}(1)^{2}$ & \multirow{2}{*}{$\begin{array}{l}(\mathbf{1}, \mathbf{1})_{3,0}=e^{T} v \\
(\mathbf{2 6}, \mathbf{1})_{-1,-1}=e^{-T}(\mathbf{2 6}, \mathbf{1})_{2,-1} \\
(\mathbf{1}, \mathbf{2})_{0,0}=e^{-T}(\mathbf{1}, \mathbf{2})_{3,0} \\
(\mathbf{1}, \mathbf{1})_{-1,2}=e^{-T}(\mathbf{1}, \mathbf{1})_{2,2}\end{array}$} \\
\hline & $\begin{array}{c}\frac{1}{16}(\mathbf{2 8}, \mathbf{2}, \mathbf{2})+ \\
\frac{1}{2}(\mathbf{2 8}, \mathbf{1}, \mathbf{2})+2(\mathbf{1}, \mathbf{2} ; \mathbf{1})\end{array}$ & $\begin{array}{c}\frac{1}{8}\left[(\mathbf{2 6}, \mathbf{2})_{1,1}+(\mathbf{1}, \mathbf{2})_{2 \pm 1,-1 \pm 1}\right] \\
+\frac{7}{8}\left[(\mathbf{2 6}, \mathbf{1})_{2,-1}+(\mathbf{1}, \mathbf{1})_{2,2}\right] \\
+2(\mathbf{1}, \mathbf{2})_{3,0} \\
\end{array}$ & \\
\hline \multirow{2}{*}{$\begin{array}{c}2 \mathrm{~B} \rightarrow 2 \mathrm{BI} \\
\langle(\mathbf{1}, \mathbf{3 2})\rangle \neq 0\end{array}$} & $\mathrm{SO}(20) \times \mathrm{SO}(12)$ & $\mathrm{SO}(20) \times \mathrm{U}(6)$ & \multirow{2}{*}{$\begin{array}{l}(\mathbf{1}, \mathbf{1})_{3}=e^{T} v \\
(\mathbf{1}, \mathbf{1 5})_{-1}=e^{-T}(\mathbf{1}, \mathbf{1 5})_{2}\end{array}$} \\
\hline & $\frac{1}{16}(\mathbf{2 0}, \mathbf{1 2})+\frac{1}{2}(\mathbf{1}, \mathbf{3 2})$ & $\frac{1}{8}(\mathbf{2 0}, \mathbf{6})_{1}+\frac{7}{8}(\mathbf{1}, \mathbf{1 5})_{2}$ & \\
\hline \multirow{2}{*}{$\begin{array}{c}2 \mathrm{C} \rightarrow 2 \mathrm{CI} \\
\left\langle(\mathbf{1 6})_{-3}\right\rangle \neq 0\end{array}$} & $\mathrm{U}(16)$ & $\mathrm{SU}(15) \times \mathrm{U}(1)^{2}$ & \multirow{2}{*}{$\begin{array}{l}(\mathbf{1})_{-3,0}=e^{T} v \\
(\mathbf{1 5})_{-1,-4}=e^{T}(\mathbf{1 5})_{2,-4}\end{array}$} \\
\hline & $\frac{1}{8}(\mathbf{1 2 0})_{2}+(\mathbf{1 6})_{-3}$ & $\begin{array}{c}\frac{1}{8}\left[(\mathbf{1 0 5})_{1,2}+(\mathbf{1 5})_{-1,6}\right] \\
+\frac{7}{8}(\mathbf{1 5})_{2,-4}\end{array}$ & \\
\hline
\end{tabular}

Table 7: Details of the matching of $\mathrm{SO}(32) \mathbb{C}^{2} / \mathbb{Z}_{2}$ models at the spectra level. For each resolution, treated on a separate row, we list the orbifold spectrum (second column), the resolution spectrum (third column) and the field redefinitions (fourth column) necessary to match the two spectra. As the blow-up induces a gauge symmetry breaking the orbifold states are branched accordingly, where we do not list those of the adjoint. Afterwards, we make a convenient U(1)basis change. Finally, the field redefinition clarifies the matching between the orbifold and resolution states.

\subsection{Flatness of the zero mode}

The flatness study of the blow-up mode in the $6 \mathrm{D}$ case is different from the $4 \mathrm{D}$ case due to the difference in the structure of the scalar potentials. In the $4 \mathrm{D}$ case the potential for the scalars in the chiral multiplets is derived from the gauge interactions (D-term potential) and from the superpotential (F-term potential). In the $6 \mathrm{D}$ case the whole potential for the scalars in the hyper multiplets is encoded in the gauge interactions. Indeed, the scalars $\Phi_{i}$ can be organized into doublets of a global SU(2) symmetry. The D-terms are defined as

$$
D^{a, \rho}=\sum \Phi_{i, M}^{*} \sigma_{M N}^{\rho} t^{a, i j} \Phi_{j, N}
$$

where $\sigma^{\rho}$ are the three Pauli matrices related to the global $\mathrm{SU}(2)$ and $t^{a}$ denotes a generator of the gauge interactions. Then, the scalar potential is just $V=D^{2}$.

A detailed study of such a potential was given in [20]. There it was shown that the D-term related to the U(1) symmetry, under which the blow-up mode is charged, cannot be zero in case a single blow-up mode is introduced. On the contrary, as argued in [20], flatness is always ensured in case more than a single mode is switched on, but not at the same fixed point, i.e. in case we have a mutual blow-up of more than one singularity.

\subsection{Anomaly cancellation}

Let's begin by studying the anomaly cancellation for the resolved models by integrating the $10 \mathrm{D}$ anomaly polynomial over the resolution. The resulting $6 \mathrm{D}$ anomaly polynomial 


$$
\hat{I}_{8}=X_{0,4} \cdot X_{4}^{u n i}+X_{2}^{n o n} \cdot X_{6}^{n o n} \equiv \hat{I}_{8}^{u n i}+\hat{I}_{8}^{n o n}
$$

where

$$
\begin{aligned}
X_{0,4} & =\operatorname{tr} R^{2}-\operatorname{tr}(i F)^{2} \\
X_{4}^{u n i} & =-\frac{1}{96}\left[\operatorname{Tr}\left[\left(\frac{1}{8} H_{V}^{2}-\frac{3}{40}\right)(i F)^{2}\right]-\frac{1}{4 \cdot(30)^{2}}\left(\operatorname{Tr}\left(H_{V} i F\right)\right)^{2}-\frac{3}{8} \operatorname{tr} R^{2}\right], \\
X_{2}^{\text {non }} & =-2 \operatorname{tr}\left[H_{V} i F\right] \\
X_{6}^{\text {non }} & =-\frac{1}{192}\left[\frac{1}{6} \operatorname{Tr}\left(H_{V}(i F)^{3}\right)-\frac{1}{120} \operatorname{Tr}\left(H_{V} i F\right)\left[\frac{1}{15} \operatorname{Tr}(i F)^{2}+\operatorname{tr} R^{2}\right]\right] .
\end{aligned}
$$

The generic polynomials given above are computed for the three $\mathbb{C}^{2} / \mathbb{Z}_{2}$ models, see Table 8 . The structure of the two terms is different, indicating different diagrams as source for the anomaly, and slightly different Green-Schwarz mechanisms. Indeed, an anomaly term factorized as $X_{2} \times X_{6}$ is canceled either by a scalar axion having an anomalous variation, or, in the dual picture, by a four-form axion. In both cases, this extra degree of freedom can be reabsorbed, fixing the gauge, into the longitudinal component of a massive vector boson, in a way similar to the Higgs mechanism or to the standard 4D Green-Schwarz mechanism. Instead, an anomaly term factorized as $X_{4} \times X_{4}$ is canceled by a two-form axion.

For the heterotic orbifold models the anomaly polynomial is always of the form $X_{4} \times X_{4}$ (see Table 8 for the explicit form for the three $\mathbb{C}^{2} / \mathbb{Z}_{2}$ models), and the GreenSchwarz mechanism involves a single "axion", i.e. the $6 \mathrm{D}$ components of the untwisted $B$-field. So, as in the 4D case, a matching between the anomaly cancellation mechanisms requires to take into account the field redefinitions that have to be performed during the blowing-up procedure. Again, a relation between the anomalies on the orbifold and on the resolution has to hold,

$$
\hat{I}_{8}^{\text {het }}+\hat{I}_{8}^{\text {red }}=\hat{I}_{8}^{\text {blow }}=\hat{I}_{8}^{u n i}+\hat{I}_{8}^{\text {non }} .
$$

One observes that $\hat{I}_{8}^{r e d}$ is factorized as $X_{2} \times X_{6}$. Thus, the corresponding anomaly cancellation mechanism induces a mass for the anomalous $\mathrm{U}(1)$. This is in agreement with the fact that, from the orbifold perspective, the blow-up corresponds to a Higgs mechanism giving a mass to the broken $\mathrm{U}(1)$.

About the matching of the axions and anti-symmetric tensor fields, things are less straightforward, since in 6D the dual of $b_{2}^{\text {blow }}$ cannot be interpreted as a massless scalar. Indeed, the matching leads to a relation among the B-fields $b_{2}^{\text {blow }} \equiv b_{2}$ and $b_{2}^{\text {orb }} \equiv h_{2}^{\text {het }}$, and the axions $a^{\text {md }}$ and $a^{T}$, which reads as

$$
b_{2}^{\text {blow }} X_{0,4}+a^{m d} X_{6}^{\text {non }}=b_{2}^{\text {orb }} X_{0,4}+a^{T} X_{6}^{\text {red }} .
$$

As explained, the axions $a^{T}, a^{\text {md }}$ and the $B$-fields $h_{2}^{\text {het }}, b_{2}$ are forms of different degree, namely 0 -forms and two-forms. Hence, one would expect relations only between forms of the same degree, i.e. $a^{T} \sim a^{m d}$ and $h_{2}^{\text {het }} \sim b_{2}$. This would require also $X_{4}^{u n i} \sim X_{4}^{\text {het }}$ 


\begin{tabular}{|c||c|}
\hline & anomaly polynomials \\
\hline \hline \multirow{2}{*}{$2 \mathrm{AI}$} & $\hat{I}_{8}^{\text {het }}=\frac{1}{2^{8}}\left[\left(i F_{26}\right)^{2}+2\left(i F_{2}\right)^{2}+12(i F)^{2}+6\left(i F^{\prime}\right)^{2}-R^{2}\right]$ \\
& {$\left[-2\left(i F_{26}\right)^{2}+12\left(i F_{2}\right)^{2}+72(i F)^{2}+84\left(i F^{\prime}\right)^{2}+192(i F)\left(i F^{\prime}\right)-R^{2}\right]$} \\
& $\hat{I}_{8}^{\text {uni }}=\frac{1}{2^{8}}\left[\left(i F_{26}\right)^{2}+2\left(i F_{2}\right)^{2}+12(i F)^{2}+6\left(i F^{\prime}\right)^{2}-R^{2}\right]$ \\
& {$\left[-2\left(i F_{26}\right)^{2}+12\left(i F_{2}\right)^{2}+264(i F)^{2}+84\left(i F^{\prime}\right)^{2}-192(i F)\left(i F^{\prime}\right)-R^{2}\right]$} \\
& $\hat{I}_{8}^{\text {non }}=\frac{1}{8}(i F)\left\{144(i F)^{3}+24\left[(i F)+\left(i F^{\prime}\right)\right]\left(i F_{2}\right)^{2}-144\left[(i F)-\left(i F^{\prime}\right)\right](i F)\left(i F^{\prime}\right)-3(i F) R^{2}\right\}$ \\
\hline \hline \multirow{2}{*}{ 2BI } & $\hat{I}_{8}^{\text {het }}=\frac{1}{2^{8}}\left[\left(i F_{20}\right)^{2}+2\left(i F_{6}\right)^{2}+12(i F)^{2}-R^{2}\right]\left[-2\left(i F_{20}\right)^{2}+12\left(i F_{6}\right)^{2}+72(i F)^{2}-R^{2}\right]$ \\
& $\hat{I}_{8}^{\text {uni }}=\frac{1}{2^{8}}\left[\left(i F_{20}\right)^{2}+2\left(i F_{6}\right)^{2}+12(i F)^{2}-R^{2}\right]\left[-2\left(i F_{20}\right)^{2}+12\left(i F_{6}\right)^{2}+72(i F)^{2}-R^{2}\right]$ \\
& $\hat{I}_{8}^{\text {non }}=\frac{1}{8}(i F)\left\{48(i F)^{3}+8\left(i F_{6}\right)^{3}+24(i F)\left(i F_{6}\right)^{2}-3(i F) R^{2}\right\}$ \\
\hline \hline \multirow{2}{*}{ 2CI } & $\hat{I}_{8}^{\text {het }}=\frac{1}{2^{8}}\left[2\left(i F_{15}\right)^{2}+12(i F)^{2}+80\left(i F^{\prime}\right)^{2}-R^{2}\right]\left[72(i F)^{2}+480(i F)\left(i F^{\prime}\right)+800\left(i F^{\prime}\right)^{2}-R^{2}\right]$ \\
& $\hat{I}_{8}^{\text {uni }}=\frac{1}{2^{8}}\left[2\left(i F_{15}\right)^{2}+12(i F)^{2}+80\left(i F^{\prime}\right)^{2}-R^{2}\right]\left[72(i F)^{2}-480(i F)\left(i F^{\prime}\right)+800\left(i F^{\prime}\right)^{2}-R^{2}\right]$ \\
& $\hat{I}_{8}^{\text {non }}=\frac{1}{8}(i F)\left\{48(i F)^{3}-1440\left(i F^{\prime}\right)^{3}+4\left(i F_{15}\right)^{3}+6\left[(i F)+2\left(i F^{\prime}\right)\right]\left(i F_{15}\right)^{2}\right.$ \\
& $\left.\left.-360\left[(i F)-4\left(i F^{\prime}\right)\right](i F)\left(i F^{\prime}\right)-3 i F\right) R^{2}\right\}$ \\
\hline
\end{tabular}

Table 8: We give the details of the anomaly cancellation in the blow-up of the various $\mathbb{C}^{2} / \mathbb{Z}_{2}$ $\mathrm{SO}(32)$ orbifold models. For each blow-up we list the orbifold anomaly polynomial $\hat{I}_{8}^{\text {het }}$ after the gauge symmetry breaking and the resolution anomaly polynomial, split into the universal $\hat{I}_{8}^{u n i}$ and non-universal $\hat{I}_{8}^{\text {non }}$ parts. Traces are again implicit.

and $X_{6}^{\text {red }} \sim X_{6}^{\text {non }}$, what is in general not true, see table 8 , Only model 2BI fulfills this condition, and in such a case we deduce

$$
b_{2}=h_{2}^{h e t}, \quad a^{m d}=-8 a^{T} .
$$

For models 2AI and 2CI, instead, both $X_{4}^{\text {uni }}, X_{4}^{\text {het }}$ and $X_{6}^{\text {red }}, X_{6}^{\text {non }}$ are not proportional, and more work is needed in order to relate the axions and $B$-fields. We address this issue in the following paragraphs.

Let us begin the discussion by listing the gauge transformations of the axions and $B$-fields

$$
\begin{array}{rll}
\text { Orbifold } & : & \delta_{\Lambda} h_{2}^{\text {het }} \nearrow X_{0,4}, \quad \delta_{\Lambda} a^{T} \nearrow 3(i F) ; \\
\text { Resolution } & : & \delta_{\Lambda} b_{2} \nearrow X_{0,4}, \quad \delta_{\Lambda} a^{m d} \nearrow X_{2}^{\text {non }},
\end{array}
$$

where the arrow $\nearrow$ indicates that the descent equations have to be used, and $\delta_{\Lambda}$ denotes a combined gauge and Lorentz transformation. The gauge transformation of the $B$-fields is model-independent and fixed, on both the orbifold and the resolution side. Hence, we have to change our description of the physical degrees of freedom to overcome this rigidity of (69) to be able to formulate relations between the fields on both sides. This can be achieved by passing to the dual forms, denoted by the two-forms $\tilde{h}_{2}^{\text {het }}, \tilde{b}_{2}$ and the four-forms $\tilde{a}_{4}:=\tilde{a}_{4}^{T}, \tilde{c}_{4}:=\tilde{a}_{4}^{\mathrm{md}}$. The duality transformation interchanges couplings 
and gauge transformations (on the level of the anomaly polynomial). Thus, the gauge transformations read as

$$
\begin{array}{rll}
\text { Orbifold } & : & \delta_{\Lambda} \tilde{h}_{2}^{\text {het }} \nearrow X_{4}^{\text {het }}, \quad \delta_{\Lambda} \tilde{a}_{4} \nearrow X_{6}^{\text {red }} ; \\
\text { Resolution } & : & \delta_{\Lambda} \tilde{b}_{2} \nearrow X_{4}^{\text {uni }}, \quad \delta_{\Lambda} \tilde{c}_{4} \nearrow X_{6}^{\text {non }},
\end{array}
$$

whereas the couplings are given by the forms in (69). Precisely these gauge transformations will help us to relate the orbifold and resolution fields appropriately. We have to express $\tilde{h}_{2}^{\text {het }}$ and $\tilde{a}_{4}$ by their counterparts $\tilde{b}_{2}, \tilde{c}_{4}$ and the Abelian gauge fields, such that both sides of these expressions transform identically under gauge transformations. Hence, their gauge transformations produce the same term on the level of the anomaly polynomial and the anomaly (66) is canceled.

We now apply this method to model 2CI to deduce the axion and $B$-field relations. The gauge transformations for $\tilde{b}_{2}$ and $\tilde{h}_{2}^{\text {het }}$ are given in this case, such that the difference is just

$$
\delta_{\Lambda}\left(\tilde{b}_{2}-\tilde{h}_{2}^{\text {het }}\right) \nearrow X_{4}^{\mathrm{uni}}-X_{4}^{\mathrm{het}}=-\frac{15}{4}(i F)\left(i F^{\prime}\right),
$$

compare Table 8. Hence we can apply the descent equations to obtain the relation between the $B$-fields

$$
\tilde{b}_{2}=\tilde{h}_{2}^{\text {het }}-\frac{15}{4}(i A)\left(i A^{\prime}\right)
$$

To relate also $\tilde{c}_{4}$ and $\tilde{a}_{4}$ we make the ansatz

$$
\tilde{c}_{4}=-\frac{1}{8} \tilde{a}_{4}+\gamma\left(i F^{\prime}\right) \tilde{h}_{2}^{h e t}+\left(i A^{\prime}\right) Y_{3}
$$

for a free parameter $\gamma$ and a three-form $Y_{3}$. Using (71) we can consider the terms induced by a gauge transformation on the level of the anomaly polynomial. This yields a factorized expression, which justifies our ansatz (75)

$$
\begin{aligned}
X_{6}^{\mathrm{non}}+\frac{1}{8} X_{6}^{\mathrm{red}} & =\left(i F^{\prime}\right)\left[-\frac{5}{16} \operatorname{tr}_{\mathbf{1 5}}\left(i F_{\mathbf{1 5}}\right)^{2}-\frac{15}{8}(i F)^{2}-\frac{25}{2}\left(i F^{\prime}\right)^{2}+\frac{5}{32} \operatorname{tr} R^{2}\right] \\
& \stackrel{!}{=} \gamma\left(i F^{\prime}\right) \tilde{h}_{2}^{\text {het }}+\left(i F^{\prime}\right) Y_{4},
\end{aligned}
$$

where the explicit expressions for $X_{6}^{\text {non }}$ and $X_{6}^{\text {red }}$ and $Y_{4}:=d Y_{3}$ were used. However, naive use of the descent equations for $\left(i F^{\prime}\right)^{3}$ yields $\left(i A^{\prime}\right)\left[\left(i A^{\prime}\right)\left(i F^{\prime}\right)\right]=0$, because $i A^{\prime}$ is an Abelian gauge field. Thus, this term can not be obtained from the anomalous variation of $\left(i A^{\prime}\right) Y_{3}$ as $Y_{3}$ had to contain $\left[\left(i A^{\prime}\right)\left(i F^{\prime}\right)\right]$. Consequently, $\left(i F^{\prime}\right)^{3}$ has to be generated by the anomalous transformation of $\tilde{h}_{2}^{\text {het }}$ that is determined by $X_{4}^{\text {het }}$. We can use this observation to determine $\gamma$. Fortunately, $X_{4}^{\text {het }}$ contains a term $\frac{25}{8}\left(i F^{\prime}\right)^{2}$, see table 8 , such that we can write

$$
\begin{gathered}
X_{6}^{\text {non }}+\frac{1}{8} X_{6}^{\text {red }} \supset-\frac{25}{2}\left(i F^{\prime}\right)^{3} \stackrel{!}{=} \gamma \frac{25}{8}\left(i F^{\prime}\right)^{3} \subset \gamma\left(i F^{\prime}\right) X_{4}^{\text {het }} \\
\Longrightarrow \gamma=-4 .
\end{gathered}
$$


Now, we are able to calculate $Y_{4}$ by plugging in all results. Hence, we obtain

$$
\begin{aligned}
\left(i F^{\prime}\right) Y_{4} & =X_{6}^{\text {non }}+\frac{1}{8} X_{6}^{\text {red }}+4\left(i F^{\prime}\right) X_{4}^{\text {het }} \\
& =\left(i F^{\prime}\right)\left[-\frac{5}{16} \operatorname{tr}_{15}\left(i F_{\mathbf{1 5}}^{2}\right)-\frac{3}{4}(i F)^{2}+\frac{15}{2}(i F)\left(i F^{\prime}\right)+\frac{9}{64} \operatorname{tr} R^{2}\right] .
\end{aligned}
$$

Finally, this results can be used for the descent equations to obtain the expression for $Y_{3}$ in terms of the Chern-Simons forms for the various characteristic classes appearing in $Y_{4}$. This concludes the explicit matching of the axions and $B$-fields in (74) and (75).

The methods presented above can also be used to relate the axions and $B$-fields of model $2 \mathrm{~A}$ and its resolution 2AI. However, the inconvenient numerical effort of this calculations will prevent us from addressing this here. Let us just note that analogous relations to the ones given above can be deduced and that $\tilde{c}_{4}=-\frac{1}{8} \tilde{a}_{4}+\ldots$ occurs. Of course, these new descriptions reproduce the results given above for model $2 \mathrm{BI}$ as we can write $\tilde{h}_{2}^{\text {het }}=\tilde{b}_{2}$ and $\tilde{c}_{4}=-\frac{1}{8} \tilde{a}_{4}$. They automatically have the same gauge transformations. After dualization of these relations we recover our old result (68), because the factor $-\frac{1}{8}$ converts to -8 . Hence, it seems also for the $6 \mathrm{D}$ models to be a modelindependent statement that $a^{\mathrm{md}}=-8 a^{T}+\ldots$, although the precise relations are much more complicated.

\section{Conclusions and outlook}

We have compared heterotic string models on orbifolds with supergravity models on smooth compact spaces obtained by resolving the corresponding orbifold. Our motivation was to extend the physics of the orbifold constructions to regions in the moduli space "far" away from the orbifold point. Our main focus was on heterotic $\mathrm{E}_{8} \times \mathrm{E}_{8}^{\prime} \mathrm{su}_{-}$ pergravity models assembled on resolutions of the $T^{6} / \mathbb{Z}_{3}$ orbifold, allowing for Wilson lines to be present. To prepare for this analysis we considered models on resolutions of the non-compact orbifold $\mathbb{C}^{3} / \mathbb{Z}_{3}$ before turning to the compact case. We achieved full agreement between orbifold and resolved models, at the level of gauge interactions, massless spectrum and anomaly cancellation.

First, we reviewed the construction of the non-compact $\mathbb{C}^{3} / \mathbb{Z}_{3}$ resolution, equipped with a gauge flux, that we completely classified in case of an $\mathrm{U}(1)$ bundle background. Then, on the level of $\mathrm{E}_{8} \times \mathrm{E}_{8}^{\prime}$ heterotic supergravity, we matched each $\mathbb{C}^{3} / \mathbb{Z}_{3}$ resolution model, built by employing Abelian gauge bundle backgrounds, with an orbifold model. This extends the results of [15] and [17] in the $\mathrm{SO}(32)$ context. We achieved the matching by single-vev blow-ups of the orbifold model, i.e. by giving a vev to a single twisted state along an F-flat direction of the potential. We emphasized that a single orbifold model has different blow-ups, if multiple twisted states are present.

After this we investigated the matching in detail. We demonstrated the fundamental importance of the blow-up mode to obtain full agreement of the spectra as well as the anomaly cancellation mechanisms in both theories. First, we used the blow-up mode 
to perform field redefinitions of the matter fields on the orbifold, so that the massless spectrum, including the U(1) charges, coincided with the one on the resolution. Then, we showed how the Higgs mechanism caused by the vev of the blow-up mode relates the maximally two anomalous U(1)'s on the resolution to the potential anomalous $\mathrm{U}(1)$ of heterotic orbifolds. A detailed analysis of the identification of the axions entering anomaly cancellation was performed: In the resolved models two axions take part in the cancellation of the two anomalous U(1)'s. Both are among the three zero-modes of the $10 \mathrm{D}$ anti-symmetric tensor field $B_{M N}$. Two zero-modes fill the whole internal space and are just constant in the blow-down limit. But only one of those has an anomalous variation and therefore corresponds to a 4D axion. From the orbifold perspective this untwisted state can be identified as the model-independent axion. The third zero-mode of $B_{M N}$ is peaked around the singularity, hence this model-dependent axion should be identified with a twisted state. It is precisely the counterpart of the blow-up mode on the orbifold. All this shows that the blow-up mode is the crucial ingredient in the matching between the orbifold and resolution models.

Since we matched SUSY orbifold models with SUSY resolved models, it was decisive to ensure F- and D-flatness of the blow-up mode. This could always be achieved up to a single D-term: precisely the one corresponding to the $\mathrm{U}(1)$ gauge symmetry that is anomalous on the resolution but not on the orbifold. This is to be expected, because when a U(1) anomaly is cancelled via the Green-Schwarz mechanism, a Fayet-Iliopoulos term is generated in the potential. The resolution model accommodates two anomalous U(1)'s and two FI terms, while the orbifold model has only a single anomalous U(1) and a single FI term. From the orbifold perspective, the additional FI term on the resolution is induced by the vev of the blow-up mode.

Next we focused on the central theme of this work, the study of resolution models of the compact $T^{6} / \mathbb{Z}_{3}$ orbifold. We resolved it by replacing each of the 27 singularities with a copy of the smooth space used in the non-compact case. In the transition to the compact case the superpotential is modified: new couplings arise among states localized in different fixed points, as well as among states at the same fixed point. Nevertheless switching on the same blow-up mode at all fixed points is still F-flat. This demonstrates that $T^{6} / \mathbb{Z}_{3}$ orbifold models in the absence of Wilson line can be blown up by simply joining 27 identical copies of the $\mathbb{C}^{3} / \mathbb{Z}_{3}$ resolution.

Discrete Wilson lines on compact orbifolds constitute a crucial ingredient of heterotic orbifold model building. Therefore, reproducing them on smooth spaces provides a definite step forward, towards the construction of realistic models in blow-up. On a compact resolution, discrete Wilson lines correspond to the possibility of having different gauge fluxes wrapping different cycles of resolved singularities. In the large volume limit, or, equivalently, in the blow-down limit, they are identified with the transition functions connecting patches surrounding different resolved singularities. We have demonstrated how to compute the resulting gauge group and matter spectrum in the presence of such transition functions; they generically lead to more gauge symmetry breaking than discrete Wilson lines on orbifolds. In particular, we can have transition functions that correspond to trivial discrete Wilson lines from the orbifold perspective, but that still induce some gauge symmetry breaking in the resolved models. Then, we investigated the 
structure of anomaly cancellation in the presence of discrete Wilson lines. Contrarily to the non-compact case, more than two anomalous $\mathrm{U}(1)$ 's may be present.

As an application of our general principles, we considered the resolution of a semirealistic $T^{6} / \mathbb{Z}_{3}$ MSSM model studied in [2]. We found that no complete blow-up is possible using $\mathrm{U}(1)$ fluxes without breaking the hypercharge of the model.

Finally, we also considered the $\mathbb{C}^{2} / \mathbb{Z}_{2}$ orbifold, in the same spirit as in the $\mathbb{C}^{3} / \mathbb{Z}_{3}$ case. We reviewed the matching of the spectra and the computation of anomaly polynomials done in [20], showing how to match, via field redefinitions, the blown-up and resolved spectra at the level of both non-Abelian and Abelian charges, and thus how to match the anomaly cancellation mechanism, completing the results of [20]. The corresponding identification of axions and anti-symmetric tensor fields is much more cumbersome than in the $\mathbb{C}^{3} / \mathbb{Z}_{3}$ case; we explained details of the matching in one specific example.

As mentioned above, our main motivation was to extend the power of the orbifold construction to regions of the moduli space where direct string quantization is very difficult to perform. This is crucial if we want to address issues like moduli stabilization or the study of the "landscape" of heterotic models. Moreover, this is essential when the orbifold model is driven away from the orbifold point by a Fayet-Iliopoulos term corresponding to an anomalous U(1). Since many resolved models constructed in this work contain anomalous U(1)'s, they do not provide stable endpoints of such flows. As we indicated, stable vacua can be obtained by vevs at different fixed points conspiring to lead to vanishing D-terms, or by multiple vevs at some fixed points. We believe that stable points in the moduli space with multiple vevs can be brought forth by smooth non-Abelian flux compactifications.

We found that many of the blown-up models can be reproduced as resolved models with $U(1)$ fluxes. But there is also a large class of orbifold blow-ups that do not lead to Abelian bundles. Of course, the "standard embedding" provides an example of a nonAbelian SU(3) background. However, there should be many non-Abelian flux models induced by giving vevs to more than one twisted states in the blow-up procedure. It would be interesting to understand, what background flux can be reproduced by such multiple vevs. We hope that the classification and matching we performed, will prove helpful to determine the topological properties of the required non-Abelian bundles.

\section{Acknowledgments}

We are grateful to Kang-Sin Choi, Arthur Hebecker, Christoff Lüdeling, Hans Peter Nilles, Saúl Ramos-Sánchez, Michael Ratz and Michael Schmidt for discussions and comments. This work was partially supported by the European Union 6th framework program MRTN-CT-2004-503069 "Quest for unification", MRTN-CT-2004-005104 "ForcesUniverse", MRTN-CT-2006-035863 "UniverseNet" and SFB-Transregio 33 "The Dark Universe" by Deutsche Forschungsgemeinschaft (DFG). The work of MT is supported by the European Community through the contract N 041273 (Marie Curie Intra-European Fellowships), he would like to thank the Institute for Theoretical Physics of Heidelberg 
for hospitality during the completion of the work.

\section{References}

[1] L. J. Dixon, J. A. Harvey, C. Vafa and E. Witten, "Strings On Orbifolds," Nucl. Phys. B 261 (1985) 678;

"Strings On Orbifolds. 2," Nucl. Phys. B 274 (1986) 285.

[2] L. E. Ibáñez, J. E. Kim, H. P. Nilles and F. Quevedo, "Orbifold Compactifications With Three Families Of SU(3) X SU(2) X U(1) ${ }^{N}$," Phys. Lett. B 191 (1987) 282.

[3] Y. Kawamura, "Triplet-doublet splitting, proton stability and extra dimension," Prog. Theor. Phys. 105, 999 (2001) arXiv:hep-ph/0012125];

G. Altarelli and F. Feruglio, "SU(5) grand unification in extra dimensions and proton decay," Phys. Lett. B 511, 257 (2001) arXiv:hep-ph/0102301];

L. J. Hall and Y. Nomura, "Gauge unification in higher dimensions," Phys. Rev. D 64, 055003 (2001) arXiv:hep-ph/0103125;

A. Hebecker and J. March-Russell, "A minimal S(1)/(Z(2) x Z'(2)) orbifold GUT," Nucl. Phys. B 613, 3 (2001) |arXiv:hep-ph/0106166];

T. Asaka, W. Buchmüller and L. Covi, "Gauge unification in six dimensions," Phys. Lett. B 523, 199 (2001) |arXiv:hep-ph/0108021];

L. J. Hall, Y. Nomura, T. Okui and D. R. Smith, "SO(10) unified theories in six dimensions," Phys. Rev. D 65, 035008 (2002) arXiv:hep-ph/0108071.

[4] T. Kobayashi, S. Raby and R. J. Zhang, "Searching for realistic 4d string models with a Pati-Salam symmetry: Orbifold grand unified theories from heterotic string compactification on a Z(6) orbifold," Nucl. Phys. B 704, 3 (2005) arXiv:hep-ph/0409098;

"Constructing 5d orbifold grand unified theories from heterotic strings," Phys. Lett. B 593, 262 (2004) arXiv:hep-ph/0403065].

[5] S. Förste, H. P. Nilles, P. K. S. Vaudrevange and A. Wingerter, "Heterotic brane world," Phys. Rev. D 70, 106008 (2004) arXiv:hep-th/0406208.

[6] A. Hebecker and M. Trapletti, "Gauge unification in highly anisotropic string compactifications," Nucl. Phys. B 713, 173 (2005) arXiv:hep-th/0411131.

[7] W. Buchmüller, K. Hamaguchi, O. Lebedev and M. Ratz, "Supersymmetric standard model from the heterotic string," Phys. Rev. Lett. 96, 121602 (2006) arXiv:hep-ph/0511035.

[8] J. E. Kim and B. Kyae, "Flipped SU(5) from Z(12-I) orbifold with Wilson line," Nucl. Phys. B 770 (2007) 47 [arXiv:hep-th/0608086].

[9] W. Buchmüller, K. Hamaguchi, O. Lebedev and M. Ratz, "Supersymmetric standard model from the heterotic string. II," Nucl. Phys. B 785, 149 (2007) 
arXiv:hep-th/0606187];

O. Lebedev, H. P. Nilles, S. Raby, S. Ramos-Sánchez, M. Ratz, P. K. S. Vaudrevange and A. Wingerter, "A mini-landscape of exact MSSM spectra in heterotic orbifolds," Phys. Lett. B 645 (2007) 88 arXiv:hep-th/0611095.

[10] A. E. Faraggi, D. V. Nanopoulos and K. j. Yuan, "A Standard Like Model in the 4D Free Fermionic String Formulation," Nucl. Phys. B 335, 347 (1990);

A. E. Faraggi, "A New standard - like model in the four-dimensional free fermionic string formulation," Phys. Lett. B 278, 131 (1992);

G. B. Cleaver, A. E. Faraggi and D. V. Nanopoulos, "String derived MSSM and M-theory unification," Phys. Lett. B 455, 135 (1999) arXiv:hep-ph/9811427.

[11] R. Blumenhagen, B. Kors, D. Lüst, and T. Ott "The standard model from stable intersecting brane world orbifolds" Nucl. Phys. B616 (2001) 3-33 [hep-th/0107138]. M. Cvetič, G. Shiu, and A. M. Uranga "Chiral four-dimensional N = 1 supersymmetric type IIA orientifolds from intersecting D6-branes" Nucl. Phys. B615 (2001) 3-32 [hep-th/0107166].

[12] T. P. T. Dijkstra, L. R. Huiszoon, and A. N. Schellekens "Supersymmetric standard model spectra from rcft orientifolds" Nucl. Phys. B710 (2005) 3-57 [hepth/0411129]. supersymmetric standard model spectra from orientifolds of gepner models" Phys. Lett. B609 (2005) 408-417 [hep-th/0403196].

[13] V. Braun, Y.-H. He, B. A. Ovrut, and T. Pantev "A heterotic standard model" Phys. Lett. B618 (2005) 252-258 [hep-th/0501070]. "A standard model from the $\mathrm{E}(8)$ x E(8) heterotic superstring" JHEP 06 (2005) 039 [hep-th/0502155].

[14] P. S. Aspinwall, "Resolution of orbifold singularities in string theory," arXiv:hep-th/9403123;

V. Bouchard, "Toric geometry and string theory," arXiv:hep-th/0609123. M. A. Walton, "The Heterotic String On The Simplest Calabi-Yau Manifold And Its Orbifold Limits," Phys. Rev. D 37 (1988) 377.

[15] S. Groot Nibbelink, M. Trapletti and M. Walter, "Resolutions of $\mathrm{C}^{* *} \mathrm{n} / \mathrm{Z}(\mathrm{n})$ orbifolds, their U(1) bundles, and applications to string model building," JHEP 0703, 035 (2007) arXiv:hep-th/0701227].

[16] S. Hamidi and C. Vafa, "Interactions on Orbifolds," Nucl. Phys. B 279 (1987) 465; L. J. Dixon, D. Friedan, E. J. Martinec and S. H. Shenker, "The Conformal Field Theory Of Orbifolds," Nucl. Phys. B 282 (1987) 13.

[17] S. Groot Nibbelink, H. P. Nilles and M. Trapletti, "Multiple anomalous U(1)s in heterotic blow-ups," Phys. Lett. B 652, 124 (2007) arXiv:hep-th/0703211].

[18] S. Groot Nibbelink, T. W. Ha and M. Trapletti, "Toric Resolutions of Heterotic Orbifolds," arXiv:0707.1597 [hep-th]. 
[19] J. Giedt "Z(3) orbifolds of the $\mathrm{SO}(32)$ heterotic string: 1 Wilson line embeddings" Nucl. Phys. B671 (2003) 133-147 [hep-th/0301232];

K.-S. Choi, S. Groot Nibbelink, and M. Trapletti "Heterotic SO(32) model building in four dimensions" JHEP 12 (2004) 063 [hep-th/0410232];

H. P. Nilles, S. Ramos-Sánchez, P. K. S. Vaudrevange, and A. Wingerter "Exploring the SO(32) heterotic string" JHEP 04 (2006) 050 [hep-th/0603086].

[20] G. Honecker and M. Trapletti "Merging heterotic orbifolds and K3 compactifications with line bundles," JHEP 0701 (2007) 051 [arXiv:hep-th/0612030].

[21] D. Lüst, S. Reffert, E. Scheidegger and S. Stieberger, "Resolved toroidal orbifolds and their orientifolds," arXiv:hep-th/0609014.

[22] P. Candelas, G. T. Horowitz, A. Strominger and E. Witten, "Vacuum Configurations For Superstrings," Nucl. Phys. B 258 (1985) 46.

[23] R. Blumenhagen, G. Honecker and T. Weigand, "Loop-corrected compactifications of the heterotic string with line bundles," JHEP 0506 (2005) 020 arXiv:hep-th/0504232.

[24] E. Witten, "Some Properties Of O(32) Superstrings," Phys. Lett. B 149 (1984) 351.

[25] A. Font, L. E. Ibáñez, H. P. Nilles and F. Quevedo, "Degenerate Orbifolds," Nucl. Phys. B 307 (1988) 109 [Erratum-ibid. B 310 (1988) 764].

[26] T. Kobayashi and N. Ohtsubo, "Yukawa coupling condition of Z(N) orbifold models," Phys. Lett. B 245 (1990) 441;

"Allowed Yukawa couplings of Z(N) x Z(M) orbifold models," Phys. Lett. B 262 (1991) 425;

T. Kobayashi, H. P. Nilles, F. Plöger, S. Raby and M. Ratz, "Stringy origin of non-Abelian discrete flavor symmetries," Nucl. Phys. B 768, 135 (2007) arXiv:hep-ph/0611020].

[27] O. Lebedev, H. P. Nilles, S. Raby, S. Ramos-Sánchez, M. Ratz, P. K. S. Vaudrevange and A. Wingerter, "The Heterotic Road to the MSSM with R parity," arXiv:0708.2691 [hep-th].

[28] K. S. Choi and T. Kobayashi, "Higher Order Couplings from Heterotic Orbifold Theory," arXiv:0711.4894 [hep-th].

[29] F. Buccella, J. P. Derendinger, S. Ferrara and C. A. Savoy, "Patterns Of Symmetry Breaking In Supersymmetric Gauge Theories," Phys. Lett. B 115 (1982) 375; F. Buccella, J. P. Derendinger, C. A. Savoy and S. Ferrara, "Symmetry Breaking In Supersymmetric Guts,"

[30] R. D. Peccei and H. R. Quinn, "CP Conservation In The Presence Of Instantons," Phys. Rev. Lett. 38 (1977) 1440. 
[31] R. D. Peccei and H. R. Quinn, "Constraints Imposed By CP Conservation In The Presence Of Instantons," Phys. Rev. D 16 (1977) 1791.

[32] P. Svrcek and E. Witten, "Axions in string theory," JHEP 0606 (2006) 051 arXiv:hep-th/0605206.

[33] M. B. Green and J. H. Schwarz, "Anomaly Cancellation In Supersymmetric D=10 Gauge Theory And Superstring Theory," Phys. Lett. B 149 (1984) 117.

[34] M. B. Green, J. H. Schwarz and E. Witten, "Superstring Theory, Vol.2" p.353 Cambridge University Press, (1987)

[35] L. E. Ibáñez, H. P. Nilles and F. Quevedo, "Orbifolds And Wilson Lines," Phys. Lett. B 187 (1987) 25.

[36] J. A. Casas and C. Muñoz, "Three Generation SU(3) x SU(2) x U(1)-Y x U(1) Orbifold Models Through Fayet-Iliopoulos Terms," Phys. Lett. B 209 (1988) 214.

[37] F. Gmeiner, S. Groot Nibbelink, H. P. Nilles, M. Olechowski and M. G. A. Walter, "Localized anomalies in heterotic orbifolds," Nucl. Phys. B 648 (2003) 35 arXiv:hep-th/0208146;

S. Groot Nibbelink, H. P. Nilles, M. Olechowski and M. G. A. Walter, "Localized tadpoles of anomalous heterotic U(1)'s," Nucl. Phys. B 665 (2003) 236 arXiv:hep-th/0303101.

[38] F. Plöger, S. Ramos-Sánchez, M. Ratz and P. K. S. Vaudrevange, "Mirage Torsion," JHEP 0704 (2007) 063 arXiv:hep-th/0702176. 